\title{
Master-Slave Composite Vibration Control of a Mobile Flexible Manipulator via Synchronization Optimization of Observation and Feedback
}

\author{
Jin-yong Ju $\left(\mathbb{1},{ }^{1}\right.$ Yufei Liu $\left(\mathbb{D},{ }^{1}\right.$ Honglin Kan, ${ }^{1}$ and Chunrui Zhang ${ }^{2}$ \\ ${ }^{1}$ School of Mechanical and Automotive Engineering, Anhui Polytechnic University, Wuhu 241000, China \\ ${ }^{2}$ Engineering Research and Training Center, Anhui Polytechnic University, Wuhu 241000, China \\ Correspondence should be addressed to Yufei Liu; yufeiliucumt@yahoo.com
}

Received 9 July 2019; Revised 5 November 2019; Accepted 22 November 2019; Published 20 December 2019

Academic Editor: Guido Caldarelli

Copyright (c) 2019 Jin-yong Ju et al. This is an open access article distributed under the Creative Commons Attribution License, which permits unrestricted use, distribution, and reproduction in any medium, provided the original work is properly cited.

\begin{abstract}
The elastic vibration of the flexible manipulator is the key problem to be solved before its effective application. The mobile flexible manipulator system (MFMS), under variable load conditions, is taken as the research object. Based on Lagrange's principle and the singular perturbation theory, the two-timescale subsystems dynamic models of the MFMS are constructed to set up the system payment function and the Hamiltonian function which correspond to the subsystems states and errors. Then, with the minimization of the system Hamiltonian function, the synchronization optimization of the designed two-timescale optimal observer (TSOO) and the designed optimal state feedback controller is realized, under the premise of the system stability which is verified by the Lyapunov stability criterion. Furthermore, with the contradiction between the control rapidity and the accuracy of the optimal state feedback controller considered, by combining the input shaping technology, the master-slave composite controller for the elastic vibration of the MFMS is constructed. Finally, simulation results verify the validity of the designed TSOO and the master-slave composite controller.
\end{abstract}

\section{Introduction}

The mobile manipulator system is a typical industrial robot system. Under the drive of the servomotors, the mobile manipulator can realize different industrial operations according to the design requirements. Thus, it is widely used in precision assembly, welding, handling, and other industrial occasions [1-3]. At present, the obvious disadvantage of the robot mobile manipulator system is its heavy structure. The load-to-weight ratio of the industrial robot is about $1: 10$ to $1: 30$, which is much smaller than the load capacity of a human, and there is still a lot of room for improvement from the bionics perspective [4]. Reducing the size of the robot structure and using light materials to realize the lightweight of the robot mechanical system can effectively improve its load-weight ratio, and it is also an effective way to realize the high speed and integration of the robot mobile manipulator system $[5,6]$. However, the lightening will inevitably produce flexibility, including large deformation and vibration, which will significantly affect the operational efficiency and motion accuracy of the mobile flexible manipulator system (MFMS). Therefore, it is of great significance to carry out the research on the vibration suppression of the MFMS [7-9].

The input shaping method is a typical feed-forward control technology. Because it does not need to measure the feedback error, by shaping the control signals, the control goals can be achieved. Therefore, the input shaping technology has been widely studied and applied in the elastic vibration control of the flexible manipulator. The input shaper consists of a series of pulses, and based on the principle of superposition and cancellation of the impulse response, the vibration can be effectively suppressed. Cole and Wongratanaphisan [10] designed a direct adaptive finite impulse response (FIR) input 
shaping method for the motion control, which was verified by being applied to the vibration control of a two-link rigid-flexible manipulator. In [11], a novel input shaping method was put forward for the underdamped systems, which can effectively avoid the influence of the system parameter uncertainty on the design of the conventional input shaper. Based on the input shaping method, the control strategy of the electronic throttle control, for reducing the shock and jerk characteristics of the manual transmission systems, was designed in [12]. However, the input shaper is essentially an open-loop control method, and its control effect is limited when there is external interference. For example, in the field of the industrial surface mounted technology (SMT), due to the different quality of the components, the MFMS has variable load conditions. At this time, the vibration suppression effect of the flexible manipulator cannot be achieved by using the input shaping device alone. It is necessary to optimize the conventional input shaper from the view of the control principle.

Fortunately, many scholars have proved that the input shaper can achieve better control results after introducing the feedback. With the PD feedback law combined, a modified input shaping was proposed for controlling the vibration of an experimental single-link flexible manipulator, which had better robustness [13]. Yuan and Chang [14] proposed a model reference zero vibration control law for improving the transient performance of the robot arm, and in the designed controller, the zero vibration (ZV) shaper was used as the feed-forward control and the model reference controller was applied to improving the system robustness and performance. Based on the designed integrated control strategy which was composed of the PI controller, the hysteresis compensator, the input shaper, and the smith predictor, the high-speed and highprecision tracking control of the nanopositioning stages was achieved [15]. Aiming at the double-pendulum systems, Mar et al. [16] designed a control system, with the extra insensitivity (EI) shaper and the PD controller combined, for effectively improving the system robustness under the variation in system parameters. Among many feedback control methods, owing to the ability that arbitrarily configuring the system closed-loop poles, the full-state feedback controller can significantly improve the dynamic characteristics of the controlled system, so it has attracted more and more attention $[17,18]$. On the basis of the full feedback of the system state variables, Yao and Deng [19] designed an active disturbance rejection adaptive controller for suppressing the parametric uncertainties and the uncertain nonlinearities of the hydraulic servo systems. Aiming at the Hopf bifurcation behaviour for both the single-machine and the multimachine power systems, a dynamic state feedback control law was presented in [20] and the analysis results verified its validity. From the existing research, it is seen that the prerequisite of the full-state feedback controller is that the controlled object is completely controllable and observable. For a practical system, because not all the state variables can be measured by physical sensors, thus in order to ensure the effectiveness of the full-state feedback controller, the state observer must be introduced [21-23]. However, the existing research usually designs the state observer first and then designs the full-state feedback controller. The state observer and the full-state feedback controller have a great influence on the control effect of the flexible manipulator elastic vibration. However, under the existing serial design idea, the synchronization optimization of the full-state feedback controller and the state observer cannot be realized effectively. On the other hand, the full-state feedback controller can eliminate the unpredictable and uncertain factors, so its correction action is more accurate and powerful. Unfortunately, it lacks the precaution of the open-loop control, which means that the elastic vibration of the flexible manipulator has been aroused in the control process before taking the corrective measures.

In summary, in order to make full use of the preventive effect and improve its antijamming ability of the input shaper, the master-slave composite controller for the elastic vibration of the MFMS is constructed by introducing the full-state feedback controller on the basis of the $\mathrm{ZV}$ input shaper. The structure and design flow of the master-slave composite controller are given. Furthermore, on the basis of the decomposed two-timescale subsystems model of the MFMS, the system payment function and Hamiltonian function are constructed based on the differential game theory. And under the stability premise of the closed-loop MFMS, on the premise of minimizing the system Hamiltonian function, the synchronization optimization of the state observer and the state feedback controller of the MFMS is realized. The structure of this paper is as follows. Section 2 gives the dynamic modelling and decomposition of the MFMS. The design process of the master-slave composite controller for the MFMS is shown in Section 3. Section 4 gives the simulation verification and analysis. Section 5 gives the conclusion of this paper.

\section{Dynamic Modelling and Decoupling Analysis}

The system sketch of the MFMS is shown in Figure 1. The alternating current $(\mathrm{AC})$ servomotor drives the base to move on the linear guide rail through the screw nut pair, and then the motion of the flexible manipulator is realized. During the modelling process, the assumptions are made as follows: (1) based on the Euler-Bernoulli beam model, the dynamic characteristics of the flexible manipulator are analyzed by only considering its transverse vibration in the motion direction; (2) the influence of gravity is ignored; and (3) the root of the flexible manipulator is rigidly connected to the base.

According to the assumed mode method [24, 25], the displacement of $P$ point on the flexible manipulator can be expressed as 


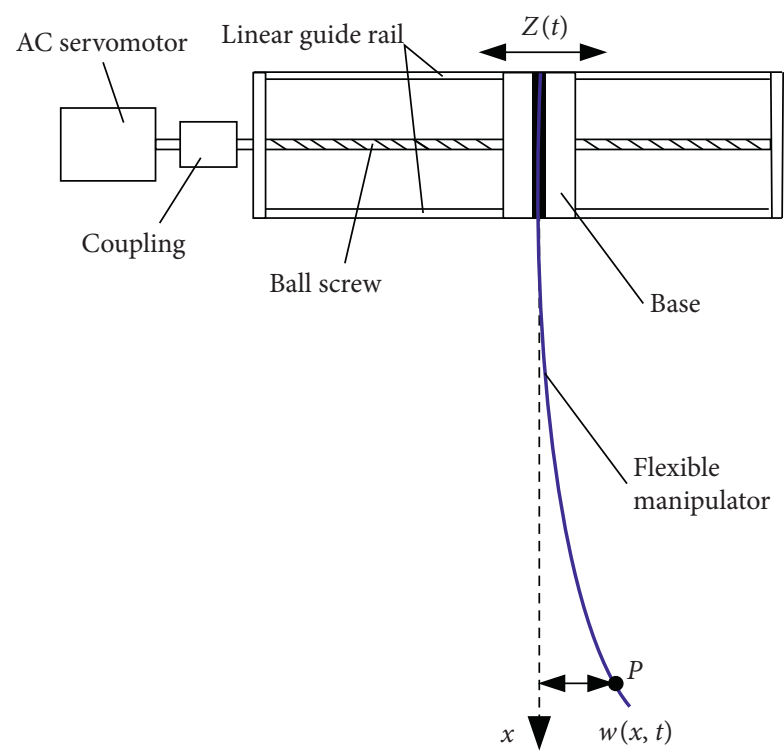

Figure 1: Schematic diagram of the MFMS.

$$
\begin{aligned}
Y(t) & =Z(t)+\omega(x, t)=Z(t)+\Phi(x) q(t) \\
& =Z(t)+\sum_{i=1}^{i \longrightarrow \infty} \phi_{i}(x) q_{i}(t),
\end{aligned}
$$

where $Z(t)$ is the base displacement, $\omega(x, t)$ is the transverse vibration displacement of the flexible manipulator, $\phi_{i}(x)$ is the $i$-th order mode function of the flexible manipulator, and $q_{i}(t)$ is the corresponding mode coordinates.

The dynamic model of the MFMS is constructed based on the energy method [26]. The kinetic energy, the potential energy, and the virtual work of the MFMS can be expressed as

$$
\begin{aligned}
& T_{e}=\frac{1}{2} m_{b} \dot{Z}^{2}(t)+\frac{1}{2} \int_{0}^{L_{b}} \rho_{b} A_{b} \dot{Y}(t)^{2} \mathrm{~d} x \\
& U_{e}=\frac{1}{2} \int_{0}^{L_{b}} E_{b} I_{b}\left[\frac{\partial^{2} \omega(x, t)}{\partial x^{2}}\right]^{2} \mathrm{~d} x \\
& \sigma W=F(t) \sigma Z(t)-\mu_{s} \int_{0}^{L_{b}} \dot{\omega}(x, t) \sigma \omega(x, t) \mathrm{d} x-v_{c} \dot{Z}(t) \sigma Z(t),
\end{aligned}
$$

where $m_{b}$ is the base quality, $\rho_{b}, A_{b}, L_{b}, E_{b}$, and $I_{b}$ are the density, the section area, the length, the modulus of elasticity, and the moment of inertia of the flexible manipulator, respectively, $F(t)$ is the driving force transmitted by the AC servomotor through the screw nut pair, $v_{c}$ represents the friction coefficient between the base and the linear guide rail, and $\mu_{s}$ is the structural damping of the flexible manipulator itself.

Then, according to Lagrange's principle [27-29], by substituting equations (2)-(4) into the second Lagrange equation, the dynamic model of the MFMS can be obtained as

$$
\begin{gathered}
{\left[\begin{array}{ll}
M_{z z} & \mathbf{M}_{z q} \\
\mathbf{M}_{z q}^{T} & \mathbf{M}_{q q}
\end{array}\right]\left[\begin{array}{l}
\ddot{Z}(t) \\
\ddot{\mathbf{q}}(t)
\end{array}\right]+\left[\begin{array}{cc}
p_{z z}(Z, \mathbf{q}, \dot{Z}, \dot{\mathbf{q}}) & 0 \\
0 & \mathbf{p}_{q q}(Z, \mathbf{q}, \dot{Z}, \dot{\mathbf{q}})
\end{array}\right]} \\
\cdot\left[\begin{array}{c}
\dot{Z}(t) \\
\dot{\mathbf{q}}(t)
\end{array}\right]+\left[\begin{array}{cc}
0 & 0 \\
0 & \mathbf{K}_{q}
\end{array}\right]\left[\begin{array}{c}
Z(t) \\
\mathbf{q}(t)
\end{array}\right]=\left[\begin{array}{c}
F(t) \\
0
\end{array}\right],
\end{gathered}
$$

where $\quad M_{z z}=m_{b}+\rho_{b} A_{b} L_{b}, \quad \mathbf{M}_{z q}=\rho_{b} A_{b} \int_{0}^{L_{b}} \boldsymbol{\Phi}(x) \mathrm{d} x$, $\mathbf{M}_{q q}=\operatorname{diag}\left[\begin{array}{llll}\rho_{b} A_{b} & \rho_{b} A_{b} & \cdots & \rho_{b} A_{b}\end{array}\right]_{i * i}, \quad p_{z z}(Z, \mathbf{q}, \dot{Z}, \dot{\mathbf{q}})$ $=v_{c}, \quad \mathbf{p}_{q q}(Z, \mathbf{q}, \dot{Z}, \dot{\mathbf{q}})=\operatorname{diag}\left[\begin{array}{llll}\mu_{s 1} & \mu_{s 2} & \cdots & \mu_{s i}\end{array}\right]_{i * i}, \quad \mathbf{K}_{q}=\operatorname{diag}$ $\left[\rho_{b} A_{b} \lambda_{1}^{2} \rho_{b} A_{b} \lambda_{2}^{2} \cdots \rho_{b} A_{b} \lambda_{i}^{2}\right]_{i * i}$ and $\lambda_{i}$ is the $i$-th natural frequency of the flexible manipulator.

According to the characteristics of the MFMS, its mass matrix is a symmetric positive definite matrix [30], which satisfies $\gamma_{\min }\|\mathbf{x}\|^{2} \leq \mathbf{x}^{T} \mathbf{M} \mathbf{x} \leq \gamma_{\max }\|\mathbf{x}\|^{2}$, where $\gamma_{\min }$ and $\gamma_{\max }$ are the minimum and maximum eigenvalues of $\mathbf{M}$. Then, the inverse matrix of $\mathbf{M}$ can be expressed as

$$
\mathbf{H}(Z, \mathbf{q})=\left[\begin{array}{ll}
H_{z z}(Z, \mathbf{q}) & \mathbf{H}_{z q}(Z, \mathbf{q}) \\
\mathbf{H}_{q z}(Z, \mathbf{q}) & \mathbf{H}_{q q}(Z, \mathbf{q})
\end{array}\right]=\left[\begin{array}{ll}
M_{z z} & \mathbf{M}_{z q} \\
\mathbf{M}_{z q}^{\mathrm{T}} & \mathbf{M}_{q q}
\end{array}\right]^{-1} .
$$

Multiplied by $\mathbf{H}(Z, \mathbf{q})$ on both sides, equation (5) can be further converted as

$$
\begin{aligned}
\ddot{Z}(t)= & H_{z z}(Z, \mathbf{q})\left[F(t)-p_{z z}(Z, \mathbf{q}, \dot{Z}, \dot{\mathbf{q}}) \dot{Z}\right] \\
& -\mathbf{H}_{z q}(Z, \mathbf{q})\left[\mathbf{p}_{q q}(S, \mathbf{q}, \dot{Z}, \dot{\mathbf{q}}) \dot{\mathbf{q}}+\mathbf{K}_{q} \mathbf{q}\right], \\
\ddot{\mathbf{q}}(t)= & \mathbf{H}_{q z}(Z, \mathbf{q})\left[F(t)-p_{z z}(Z, \mathbf{q}, \dot{Z}, \dot{\mathbf{q}}) \dot{Z}\right] \\
& -\mathbf{H}_{q q}(Z, \mathbf{q})\left[\mathbf{p}_{q q}(Z, \mathbf{q}, \dot{Z}, \dot{\mathbf{q}}) \dot{\mathbf{q}}+\mathbf{K}_{q} \mathbf{q}\right] .
\end{aligned}
$$

By introducing the singular perturbation parameter as $\varepsilon=$ $\sqrt{1 / K_{\min }}$ where $K_{\min }=\min \left(\mathbf{K}_{q}\right)$ and defining $\boldsymbol{\xi}=\mathbf{q} / \varepsilon^{2}$ and $\widetilde{\mathbf{K}}_{q}=\varepsilon^{2} \mathbf{K}_{q}$, equations (7) and (8) can be further expressed as

$$
\left\{\begin{array}{l}
\dot{x}_{1}=x_{2}, \\
\dot{x}_{2}=H_{z z}\left(x_{1}, \varepsilon^{2} \mathbf{z}_{1}\right)\left[F(t)-p_{z z}\left(x_{1}, \varepsilon^{2} \mathbf{z}_{1}, x_{2}, \varepsilon \mathbf{z}_{2}\right) x_{2}\right] \\
-\varepsilon^{2} \mathbf{p}_{q q}\left(x_{1}, \varepsilon^{2} \mathbf{z}_{1}, x_{2}, \varepsilon \mathbf{z}_{2}\right) \mathbf{z}_{2}-\mathbf{H}_{z q}\left(x_{1}, \varepsilon^{2} \mathbf{z}_{1}\right) \widetilde{\mathbf{K}}_{q} \mathbf{z}_{1},
\end{array}\right.
$$

$$
\left\{\begin{array}{l}
\dot{\mathbf{z}}_{1}=\mathbf{z}_{2}, \\
\varepsilon \dot{\mathbf{z}}_{2}=\mathbf{H}_{q z}\left(x_{1}, \varepsilon^{2} \mathbf{z}_{1}\right)\left[F(t)-p_{z z}\left(x_{1}, \varepsilon^{2} \mathbf{z}_{1}, x_{2}, \varepsilon \mathbf{z}_{2}\right) x_{2}\right] \\
-\varepsilon^{2} \mathbf{p}_{q q}\left(x_{1}, \varepsilon^{2} \mathbf{z}_{1}, x_{2}, \varepsilon \mathbf{z}_{2}\right) \mathbf{z}_{2}-\mathbf{H}_{q q}\left(x_{1}, \varepsilon^{2} \mathbf{z}_{1}\right) \widetilde{\mathbf{K}}_{q} \mathbf{z}_{1},
\end{array}\right.
$$

where $x_{1}=Z(t), x_{2}=\dot{Z}(t), \mathbf{z}_{1}=\boldsymbol{\xi}(t)$, and $\mathbf{z}_{2}=\varepsilon \dot{\xi}(t)$.

When the singular perturbation parameter equals to 0 , according to equation (10), the static vibration solution of the flexible manipulator can be obtained as

$$
\xi=\left(\widetilde{\mathbf{K}}_{q}\right)^{-1}\left[\mathbf{H}_{q q}(Z, 0)\right]^{-1}\left\{\mathbf{H}_{q z}(Z, 0)\left[F(t)-p_{s s}(Z, 0, \dot{Z}, 0) \dot{Z}\right]\right\} \text {. }
$$

Through substituting equation (11) into equation (9), the state-space equation of the slow subsystem of the MFMS can be obtained as 


$$
\left\{\begin{array}{l}
\dot{x}_{1}=x_{2} \\
\dot{x}_{2}=-M_{z z}^{-1} p_{z z}(Z, 0, \dot{Z}, 0) x_{2}+M_{z z}^{-1} F(t) .
\end{array}\right.
$$

Furthermore, for the purpose of fixing the model error between the slow subsystem and the actual MFMS, the correction parameters of the boundary layer are introduced as $\boldsymbol{\alpha}_{1}=\boldsymbol{\xi}-\overline{\boldsymbol{\xi}}=\mathbf{z}_{1}-\overline{\mathbf{z}}_{1}$ and $\boldsymbol{\alpha}_{2}=\boldsymbol{\varepsilon} \dot{\xi}=\mathbf{z}_{2}$. The fast-changing timescale is defined as $\kappa=t / \varepsilon$. Then, when $\kappa \longrightarrow 0$ near the boundary layer region, the slow variables of the MFMS can be regarded as constants. The state-space equation of the fast subsystem of the MFMS can be expressed as

$$
\left\{\begin{array}{l}
\frac{\mathrm{d} \boldsymbol{\alpha}_{1}}{\mathrm{~d} \kappa}=\boldsymbol{a}_{2}, \\
\frac{\mathrm{d} \boldsymbol{\alpha}_{2}}{\mathrm{~d} \kappa}=\mathbf{H}_{q z}\left(x_{1}, 0\right) F(t)-\mathbf{H}_{q q}\left(x_{1}, 0\right) \widetilde{\mathbf{K}}_{q} \boldsymbol{\alpha}_{1} .
\end{array}\right.
$$

In summary, equations (12) and (13) describe the twotimescale subsystems dynamic models of the MFMS. And the inputs and outputs of the original system and the twotimescale subsystems are exactly the same. The system input of the original system is the driving force $(F(t))$ transmitted by the AC servomotor through the screw nut pair, while the system inputs of the two-timescale subsystems are $F(t)$ too. The two-timescale subsystems split the outputs of the original system. The outputs of the slow subsystem are the displacement and the speed of the base, and the outputs of the fast subsystem are the state variables of the flexible manipulator vibration. Furthermore, the expression forms of the twotimescale subsystems are simpler, which is more convenient to convert to the form of the state-space equation. And it is helpful to simplify the design of the vibration controller.

\section{Master-Slave Composite Controller Design}

The master-slave composite controller for the elastic vibration control of the MFMS includes the feed-forward input shaper and the optimal state feedback controller. Considering that the existence of the time-lag link in the input shaper will affect the stability of the closed-loop MFMS, the combination mode of the input shaper outside the closed loop of the optimal state feedback controller is adopted [31, 32].

The design process of the master-slave composite controller of the MFMS is shown in Figure 2(a). Firstly, the optimal state feedback controller for the system elastic vibration is designed to determine the dominant poles of the closed-loop MFMS, which is also the design basis of the control parameters of the feed-forward input shaper. On the other hand, because the effective action premise of the optimal state feedback controller is the completely knowable of the system state variables, and thus the system state observer of the MFMS should be designed. Moreover, owing to the fact that the MFMS is a strongly coupled system, the base displacement under slow time-varying scale and the elastic vibration of the flexible manipulator under fast time-varying scale are mutually coupled. In these two-timescale subsystems, there are many problems in estimating the system physical quantities simultaneously in the same observer, such as large computation and poor real-time performance. Thus, according to the two-timescale subsystems dynamic models of the MFMS, the two-timescale optimal observer (TSOO), observing the base displacement and the vibration of the flexible manipulator, is designed. And the optimal state feedback controller based on the TSOO to realize the effective control of the flexible manipulator elastic vibration is also presented. Most importantly, based on the differential game theory $[33,34]$, the synchronous optimization of the optimal state feedback controller and the TSOO is realized. Secondly, under the action of the optimal state feedback controller, the dominant poles of the closed-loop MFMS are determined. Then, based on the principle of superposition and cancellation of the impulse response, the ZV input shaper of the closedloop MFMS is designed. Finally, according to the combination form in Figure 2(b), the optimal state feedback controller and the ZV input shaper of the closed-loop MFMS are combined as the master-slave composite controller.

Based on the two-timescale subsystems dynamic models of the MFMS, the system TSOO can be represented as

$$
\begin{aligned}
& \dot{\hat{\mathbf{x}}}=\mathbf{A}_{z} \widehat{\mathbf{x}}+\mathbf{B}_{z} F+\mathbf{K}_{z}(Z-\widehat{Z}), \\
& \dot{\hat{\boldsymbol{\alpha}}}=\mathbf{A}_{f} \widehat{\boldsymbol{\alpha}}+\mathbf{B}_{f} F+\mathbf{K}_{f}[\omega(L, t)-\widehat{\omega}(L, t)],
\end{aligned}
$$

where $\quad \mathbf{A}_{z}=\left[\begin{array}{cc}0 & 1 \\ 0 & -M_{z z}^{-1} p_{z z}(Z, 0, \dot{Z}, 0)\end{array}\right], \quad \mathbf{B}_{z}=\left[\begin{array}{c}0 \\ M_{z z}^{-1}\end{array}\right]$, $\mathbf{A}_{f}=\left[\begin{array}{cc}0 & \mathbf{I} \\ -\mathbf{H}_{q q}\left(x_{1}, 0\right) \tilde{\mathbf{K}}_{q} & 0\end{array}\right], \quad \mathbf{B}_{f}=\left[\begin{array}{c}0 \\ \mathbf{H}_{q z}\left(x_{1}, 0\right)\end{array}\right], \quad \widehat{Z} \quad$ and $\widehat{\omega}(L, t)$ are the observed values of the base displacement and vibration displacement at the end of the flexible manipulator, respectively, and $\mathbf{K}_{z}$ and $\mathbf{K}_{f}$ are the observation gains of the TSOO.

Subtracting equations (14) and (15) from equations (12) and (13), respectively, one can obtain

$$
\left\{\begin{array}{l}
\dot{\tilde{\mathbf{x}}}(t)=\mathbf{A}_{z} \widetilde{\mathbf{x}}(t)+\mathbf{u}(t) \\
\dot{\tilde{\mathbf{\alpha}}}(t)=\mathbf{A}_{f} \widetilde{\boldsymbol{\alpha}}(t)+\mathbf{v}(t)
\end{array}\right.
$$

where $\widetilde{\mathbf{x}}(t)$ and $\widetilde{\boldsymbol{\alpha}}(t)$ are the observation error of $\mathbf{x}(t)$ and $\boldsymbol{\alpha}(t)$, respectively. And the corresponding control variables can be expressed as

$$
\left\{\begin{array}{l}
\mathbf{u}(t)=-\mathbf{K}_{z} \widetilde{Z} \\
\mathbf{v}(t)=-\mathbf{K}_{f} \widetilde{\omega}(L, t)
\end{array}\right.
$$

Then, the optimal state feedback controller of the MFMS based on the observed values can be expressed as

$$
\mathbf{w}(t)=\mathbf{K}_{c} \widehat{\mathbf{e}}(t)
$$

where $\mathbf{K}_{c}$ is the control gain of the optimal state feedback controller.

In order to optimize the gains of the designed TSOO and the optimal state feedback controller, the state variables are defined as $\mathbf{e}=\left[\begin{array}{ll}\mathbf{x} & \boldsymbol{\alpha}\end{array}\right]^{\mathrm{T}}$ and the differential game model and the payment function for the closed-loop MFMS with system state variables and error are considered, which are expressed as 


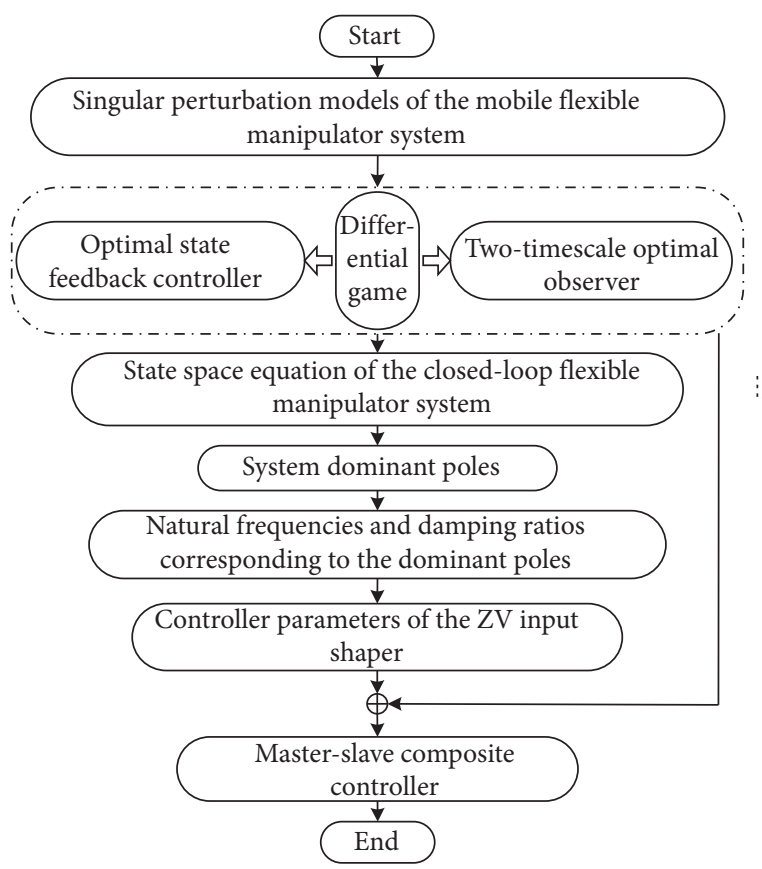

(a)

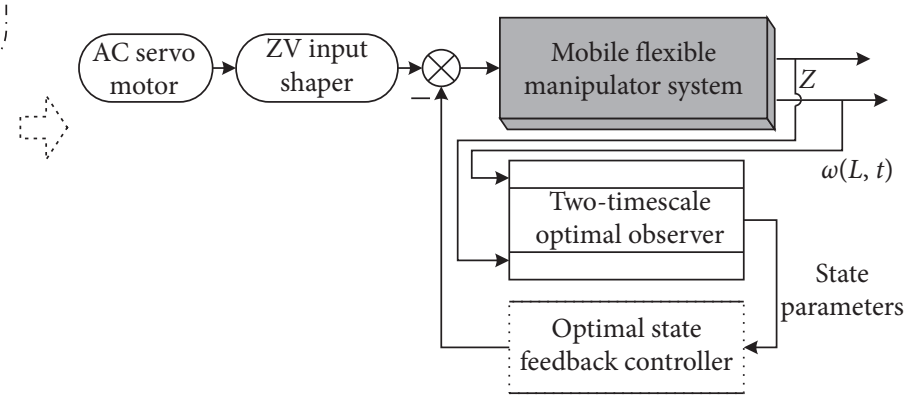

(b)

Figure 2: Design process and structure of the master-slave composite controller: (a) design process and (b) structure.

$$
\begin{aligned}
\left\{\begin{array}{l}
\dot{\mathbf{e}}(t)=\mathbf{A}_{w} \mathbf{e}(t)+\mathbf{B}_{w} \mathbf{w}(t), \\
\dot{\widetilde{\mathbf{x}}}(t)=\mathbf{A}_{z} \widetilde{\mathbf{x}}(t)+\mathbf{u}(t), \\
\dot{\tilde{\mathbf{\alpha}}}(t)=\mathbf{A}_{f} \widetilde{\mathbf{a}}(t)+\mathbf{v}(t),
\end{array}\right. \\
J(\mathbf{e}(t), \widetilde{\mathbf{x}}(t), \widetilde{\mathbf{\alpha}}(t), \mathbf{w}(t), \mathbf{u}(t), \mathbf{v}(t))=\int_{0}^{\infty}\left[\mathbf{e}^{\mathrm{T}}(t) \mathbf{Q}_{1} \mathbf{e}(t)+\widetilde{\mathbf{x}}^{\mathrm{T}}(t) \mathbf{Q}_{2} \widetilde{\mathbf{x}}(t)+\widetilde{\mathbf{a}}^{\mathrm{T}}(t) \mathbf{Q}_{3} \widetilde{\mathbf{a}}(t)+\mathbf{w}^{\mathrm{T}}(t) \mathbf{R}_{1} \mathbf{w}(t)\right. \\
\left.+\mathbf{u}^{\mathrm{T}}(t) \mathbf{R}_{2} \mathbf{u}(t)+\mathbf{v}^{\mathrm{T}}(t) \mathbf{R}_{3} \mathbf{v}(t)\right] \mathrm{d} t,
\end{aligned}
$$

where $\mathbf{A}_{w}=\left[\begin{array}{cc}\mathbf{A}_{z} & 0 \\ 0 & \mathbf{A}_{f}\end{array}\right], \mathbf{B}_{w}=\left[\begin{array}{cc}\mathbf{B}_{z} & 0 \\ 0 & \mathbf{B}_{f}\end{array}\right], \mathbf{Q}_{1}$ and $\mathbf{R}_{1}$ are symmetric positive definite matrices and the orders are $2 i+2, \mathbf{Q}_{2}$ and $\mathbf{R}_{2}$ are symmetric positive definite matrices and the orders are 2 , and $\mathbf{Q}_{3}$ and $\mathbf{R}_{3}$ are also symmetric positive definite matrices and the orders are $2 i$.

Through analyzing the closed-loop equilibrium strategy of the above differential game model, the Hamiltonian function of the closed-loop MFMS is defined as

$$
\begin{aligned}
H\left(\mathbf{e}, \widetilde{\mathbf{x}}, \widetilde{\boldsymbol{\alpha}}, \mathbf{w}, \mathbf{u}, \mathbf{v}, \mathbf{e}_{0}, \widetilde{\mathbf{x}}_{0}, \widetilde{\boldsymbol{\alpha}}_{0}\right)= & \mathbf{e}^{\mathrm{T}}(t) \mathbf{Q}_{1} \mathbf{e}(t)+\widetilde{\mathbf{x}}^{\mathrm{T}}(t) \mathbf{Q}_{2} \widetilde{\mathbf{x}}(t)+\widetilde{\boldsymbol{\alpha}}^{\mathrm{T}}(t) \mathbf{Q}_{3} \widetilde{\boldsymbol{\alpha}}(t)+\mathbf{w}^{\mathrm{T}}(t) \mathbf{R}_{1} \mathbf{w}(t) \\
& +\mathbf{u}^{\mathrm{T}}(t) \mathbf{R}_{2} \mathbf{u}(t)+\mathbf{v}^{\mathrm{T}}(t) \mathbf{R}_{3} \mathbf{v}(t) \\
& +\mathbf{e}^{\mathrm{T}}(t)\left(\mathbf{A}_{w}^{\mathrm{T}} \mathbf{P}_{1}+\mathbf{P}_{1} \mathbf{A}_{w}\right) \mathbf{e}(t)+\widetilde{\mathbf{x}}^{\mathrm{T}}(t)\left(\mathbf{A}_{z}^{\mathrm{T}} \mathbf{P}_{2}+\mathbf{P}_{2} \mathbf{A}_{z}\right) \widetilde{\mathbf{x}}(t) \\
& +\widetilde{\boldsymbol{\alpha}}^{\mathrm{T}}(t)\left(\mathbf{A}_{f}^{\mathrm{T}} \mathbf{P}_{3}+\mathbf{P}_{3} \mathbf{A}_{f}\right) \widetilde{\boldsymbol{\alpha}}(t)+\mathbf{e}^{\mathrm{T}}(t) \mathbf{P}_{1} \mathbf{B}_{w} \mathbf{w}(t)+\mathbf{w}^{\mathrm{T}}(t) \mathbf{B}_{w}^{\mathrm{T}} \mathbf{P}_{1} \mathbf{e}(t) \\
& +\widetilde{\mathbf{x}}^{\mathrm{T}}(t) \mathbf{P}_{2} \mathbf{u}(t)+\mathbf{u}^{\mathrm{T}}(t) \mathbf{P}_{2} \widetilde{\mathbf{x}}(t)+\widetilde{\boldsymbol{\alpha}}^{\mathrm{T}}(t) \mathbf{P}_{3} \mathbf{v}(t)+\mathbf{v}(t) \mathbf{P}_{3} \widetilde{\boldsymbol{\alpha}}(t) .
\end{aligned}
$$


Obviously, the existence conditions for the minimum values of the above Hamiltonian functions are as follows:

$$
\left\{\begin{array}{l}
\frac{\partial H}{\partial \mathbf{w}}=2 \mathbf{B}_{w}^{\mathrm{T}} \mathbf{P}_{1} \mathbf{e}(t)+2 \mathbf{R}_{1} \mathbf{w}(t)=0 \\
\frac{\partial H}{\partial \mathbf{u}}=2 \mathbf{P}_{2} \widetilde{\mathbf{x}}(t)+2 \mathbf{R}_{2} \mathbf{u}(t)=0 \\
\frac{\partial H}{\partial \mathbf{v}}=2 \mathbf{P}_{3} \widetilde{\boldsymbol{a}}(t)+2 \mathbf{R}_{3} \mathbf{v}(t)=0
\end{array}\right.
$$

It is seen from equation (21) that because $\partial^{2} H / \partial \mathbf{w}^{2}=2 \mathbf{R}_{1}>0, \quad \partial^{2} H / \partial \mathbf{u}^{2}=2 \mathbf{R}_{2}>0, \quad$ and $\partial^{2} H / \partial \mathbf{v}^{2}=2 \mathbf{R}_{3}>0$, the Hamiltonian function of the closedloop MFMS has a minimal value. And the control gains of the TSOO and the optimal state feedback controller for the MFMS can be calculated as

$$
\left\{\begin{array}{l}
\mathbf{w}^{\gamma}(t)=-\mathbf{R}_{1}^{-1} \mathbf{B}_{w}^{\mathrm{T}} \mathbf{P}_{1} \mathbf{e}(t) \\
\mathbf{u}^{\gamma}(t)=-\mathbf{R}_{2}^{-1} \mathbf{P}_{2} \widetilde{\mathbf{x}}(t) \\
\mathbf{v}^{\gamma}(t)=-\mathbf{R}_{3}^{-1} \mathbf{P}_{3} \widetilde{\mathbf{a}}(t)
\end{array}\right.
$$

By substituting equation (22) into equation (20), one can obtain

$$
\begin{aligned}
H\left(\mathbf{e}, \widetilde{\mathbf{x}}, \widetilde{\mathbf{a}}, \mathbf{w}^{\gamma}, \mathbf{u}^{\gamma}, \mathbf{v}^{\gamma}, \mathbf{e}_{0}, \widetilde{\mathbf{x}}_{0}, \widetilde{\mathbf{a}}_{0}\right)= & \mathbf{e}^{\mathrm{T}}(t)\left[\mathbf{A}_{w}^{\mathrm{T}} \mathbf{P}_{1}+\mathbf{P}_{1} \mathbf{A}_{w}\right. \\
& \left.-\mathbf{P}_{1} \mathbf{B}_{w} \mathbf{R}_{1}^{-1} \mathbf{B}_{w}^{\mathrm{T}} \mathbf{P}_{1}+\mathbf{Q}_{1}\right] \mathbf{e}(t) \\
& +\widetilde{\mathbf{x}}^{\mathrm{T}}(t)\left[\mathbf{A}_{z}^{\mathrm{T}} \mathbf{P}_{2}+\mathbf{P}_{2} \mathbf{A}_{z}\right. \\
& \left.-\mathbf{P}_{2} \mathbf{R}_{2}^{-1} \mathbf{P}_{2}+\mathbf{Q}_{2}\right] \widetilde{\mathbf{x}}(t) \\
& +\widetilde{\boldsymbol{a}}^{\mathrm{T}}(t)\left[\mathbf{A}_{f}^{\mathrm{T}} \mathbf{P}_{3}+\mathbf{P}_{3} \mathbf{A}_{f}\right. \\
& \left.-\mathbf{P}_{3} \mathbf{R}_{3}^{-1} \mathbf{P}_{3}+\mathbf{Q}_{3}\right] \widetilde{\mathbf{a}}(t)
\end{aligned}
$$

Then, the Riccati equations for $\mathbf{P}_{1}, \mathbf{P}_{2}$, and $\mathbf{P}_{3}$ can be determined from equation (23) as

$$
\left\{\begin{array}{l}
\mathbf{A}_{w}^{\mathrm{T}} \mathbf{P}_{1}+\mathbf{P}_{1} \mathbf{A}_{w}-\mathbf{P}_{1} \mathbf{B}_{w} \mathbf{R}_{1}^{-1} \mathbf{B}_{w}^{\mathrm{T}} \mathbf{P}_{1}+\mathbf{Q}_{1}=0 \\
\mathbf{A}_{z}^{\mathrm{T}} \mathbf{P}_{2}+\mathbf{P}_{2} \mathbf{A}_{z}-\mathbf{P}_{2} \mathbf{R}_{2}^{-1} \mathbf{P}_{2}+\mathbf{Q}_{2}=0 \\
\mathbf{A}_{f}^{\mathrm{T}} \mathbf{P}_{3}+\mathbf{P}_{3} \mathbf{A}_{f}-\mathbf{P}_{3} \mathbf{R}_{3}{ }^{-1} \mathbf{P}_{3}+\mathbf{Q}_{3}=0
\end{array}\right.
$$

The premise that the designed TSOO and the optimal state feedback controller have practical significance is that the closed-loop MFMS must be stable. Thus, in order to prove the stability of the MFMS under the action of the designed TSOO and the optimal state feedback controller, the system Lyapunov function is defined as

$$
\begin{aligned}
V(\mathbf{e}(t), \widetilde{\mathbf{x}}(t), \widetilde{\boldsymbol{\alpha}}(t))= & \mathbf{e}^{\mathrm{T}}(t) \mathbf{P}_{1} \mathbf{e}(t)+\widetilde{\mathbf{x}}^{\mathrm{T}}(t) \mathbf{P}_{2} \widetilde{\mathbf{x}}(t) \\
& +\widetilde{\boldsymbol{\alpha}}^{\mathrm{T}}(t) \mathbf{P}_{3} \widetilde{\mathbf{a}}(t) .
\end{aligned}
$$

Differentiating equation (25) with respect to time yields

$$
\begin{aligned}
\dot{V}(\mathbf{e}(t), \widetilde{\mathbf{x}}(t), \widetilde{\mathbf{a}}(t))= & \dot{\mathbf{e}}^{\mathrm{T}}(t) \mathbf{P}_{1} \mathbf{e}(t)+\mathbf{e}^{\mathrm{T}}(t) \mathbf{P}_{1} \dot{\mathbf{e}}(t) \\
& +\dot{\tilde{\mathbf{a}}}^{\mathrm{T}}(t) \mathbf{P}_{3} \widetilde{\mathbf{a}}(t)+\widetilde{\boldsymbol{\alpha}}^{\mathrm{T}}(t) \mathbf{P}_{3} \dot{\dot{\mathbf{a}}}(t) \\
& +\dot{\widetilde{\mathbf{x}}}^{\mathrm{T}}(t) \mathbf{P}_{2} \widetilde{\mathbf{x}}(t)+\widetilde{\mathbf{x}}^{\mathrm{T}}(t) \mathbf{P}_{2} \dot{\tilde{\mathbf{x}}}(t) \\
= & \mathbf{e}^{\mathrm{T}}(t)\left[\mathbf{A}_{w}^{\mathrm{T}} \mathbf{P}_{1}+\mathbf{P}_{1} \mathbf{A}_{w}\right. \\
& \left.-\mathbf{P}_{1} \mathbf{B}_{w} \mathbf{R}_{1}^{-1} \mathbf{B}_{w}^{\mathrm{T}} \mathbf{P}_{1}+\mathbf{Q}_{1}\right] \mathbf{e}(t) \\
& +\widetilde{\mathbf{x}}^{\mathrm{T}}(t)\left[\mathbf{A}_{z}^{\mathrm{T}} \mathbf{P}_{2}+\mathbf{P}_{2} \mathbf{A}_{z}\right. \\
& \left.-\mathbf{P}_{2} \mathbf{R}_{2}^{-1} \mathbf{P}_{2}+\mathbf{Q}_{2}\right] \widetilde{\mathbf{x}}(t) \\
& +\widetilde{\boldsymbol{\alpha}}^{\mathrm{T}}(t)\left[\mathbf{A}_{f}^{\mathrm{T}} \mathbf{P}_{3}+\mathbf{P}_{3} \mathbf{A}_{f}\right. \\
& \left.-\mathbf{P}_{3} \mathbf{R}_{3}^{-1} \mathbf{P}_{3}+\mathbf{Q}_{3}\right] \widetilde{\mathbf{a}}(t)-\mathbf{e}^{\mathrm{T}}(t) \mathbf{Q}_{1} \mathbf{e}(t) \\
& -\widetilde{\mathbf{x}}^{\mathrm{T}}(t) \mathbf{Q}_{2} \widetilde{\mathbf{x}}(t) \\
& -\widetilde{\boldsymbol{\alpha}}^{\mathrm{T}}(t) \mathbf{Q}_{3} B(t)-\mathbf{w}^{\mathrm{T}}(t) \mathbf{R}_{1} \mathbf{w}(t) \\
& -\mathbf{u}^{\mathrm{T}}(t) \mathbf{R}_{2} \mathbf{u}(t)-\mathbf{v}^{\mathrm{T}}(t) \mathbf{R}_{3} \mathbf{v}(t) .
\end{aligned}
$$

By substituting equation (24) into equation (26), because $\mathbf{Q}_{1}, \mathbf{Q}_{2}, \mathbf{Q}_{3}, \mathbf{R}_{1}, \mathbf{R}_{2}$, and $\mathbf{R}_{3}$ are symmetric positive definite matrices, then one can obtain

$$
\begin{aligned}
\dot{V}(\mathbf{e}(t), \widetilde{\mathbf{x}}(t), \widetilde{\mathbf{a}}(t))= & -\mathbf{e}^{\mathrm{T}}(t) \mathbf{Q}_{1} \mathbf{e}(t)-\widetilde{\mathbf{x}}^{\mathrm{T}}(t) \mathbf{Q}_{2} \widetilde{\mathbf{x}}(t) \\
& -\widetilde{\boldsymbol{a}}^{\mathrm{T}}(t) \mathbf{Q}_{3} \widetilde{\boldsymbol{\alpha}}(t)-\mathbf{w}^{\mathrm{T}}(t) \mathbf{R}_{1} \mathbf{w}(t) \\
& -\mathbf{u}^{\mathrm{T}}(t) \mathbf{R}_{2} \mathbf{u}(t)-\mathbf{v}^{\mathrm{T}}(t) \mathbf{R}_{3} \mathbf{v}(t)<0 .
\end{aligned}
$$

It is obvious from equation (27) that the closed-loop MFMS is asymptotically stable. Thus, the designed TSOO and optimal state feedback controller are feasible.

Furthermore, the dominant poles of the closed-loop MFMS are assumed to have the following characteristic polynomial, which is expressed as

$$
s^{2}+a s+b=0
$$

Then, the natural frequency and damping ratio of the closed-loop MFMS with the corresponding dominant poles can be expressed as

$$
\left\{\begin{array}{l}
w_{n}=\sqrt{b}, \\
\varsigma=\frac{a}{2 \sqrt{b}} .
\end{array}\right.
$$

The feed-forward input shaper for the elastic vibration of the closed-loop MFMS with $n$ pulses can be expressed as

$$
G_{i n}(S)=\sum_{i=1}^{n} A_{i} e^{-t_{i} S},
$$

where $A_{i}$ and $t_{i}$ are the amplitude and time delay of the $i$-th pulse of the feed-forward input shaper, respectively.

According to the control principle of the $\mathrm{ZV}$ input shaper $[35,36]$, under the action of the $\mathrm{ZV}$ input shaper, the 
elastic vibration response of the flexible manipulator should tend to zero, and the corresponding mathematical expressions can be expressed as

$$
\left\{\begin{array}{l}
\sum_{i=1}^{n} A_{i} e^{\varsigma w_{n} t_{i}} \cos \left(w_{n} \sqrt{1-\varsigma^{2}} t_{i}\right)=0, \\
\sum_{i=1}^{n} A_{i} e^{\zeta w_{n} t_{i}} \sin \left(w_{n} \sqrt{1-\varsigma^{2}} t_{i}\right)=0
\end{array}\right.
$$

In order to minimize the delay effect introduced by the $\mathrm{ZV}$ input shaper, two pulses are taken into consideration $(n=2)$, and the delay parameter of the first pulse is equal to 0 $\left(t_{1}=0\right)$. On the other hand, in order to ensure that the expected system output point can be obtained, the amplitudes of each pulse of the $\mathrm{ZV}$ input shaper should be satisfied:

$$
\sum_{i=1}^{2} A_{i}=1 .
$$

With equation (31) and equation (32) combined, the control parameters of the ZV input shaper for the closed-loop MFMS can be determined as

$$
\left\{\begin{array}{l}
A_{1}=\frac{1}{1+e^{\left(\pi / w_{n} \sqrt{1-\zeta^{2}}\right)}} \\
A_{2}=\frac{e^{\left(\pi / w_{n} \sqrt{1-\zeta^{2}}\right)}}{1+e^{\left(\pi / w_{n} \sqrt{1-\zeta^{2}}\right)}} .
\end{array}\right.
$$

\section{Simulation Verification and Analysis}

In order to verify the validity of the designed TSOO and the master-slave composite controller for the MFMS, a simulation analysis model is built by MATLAB/Simulink 2015 and the analysis verification is carried out. The physical parameters of the flexible manipulator are $L_{b}=0.4 \mathrm{~m}, \quad \rho_{b}=2030 \mathrm{~kg} / \mathrm{m}^{-3}, E=25.24 \mathrm{GPa}$, and $A_{b}=$ $1.35 E-4 \mathrm{~m}^{2}$. The structure of the flexible manipulator studied in this paper can be simplified as the EulerBernoulli beam, and based on the existing research, it is known that the first few low-order modes play a dominant role in its vibration. Then, the first two modes of the flexible manipulator are taken into consideration $[37,38]$. And according to Section 3, the observational gains of the TSOO can be determined as $\mathbf{K}_{z}=\left[\begin{array}{ll}1.5800 E 5 & 2.5567 E 3\end{array}\right]^{\mathrm{T}}$ and $\mathbf{K}_{f}=\left[\begin{array}{llll}3.2627 E 4 & 2.1494 E 3 & 0.0014 & -0.0163\end{array}\right]^{\mathrm{T}}$.

For the purpose of illustrating the effectiveness of the designed TSOO, the pole assignment method is used to design the conventional state observer (PSPO) for comparison. Under the action of the unit step excitation, the observation effects of the TSOO and the PSPO on the base displacement and speed are shown in Figures 3 and 4.

It is seen from Figure 3 that both the designed TSOO and the PSPO can effectively estimate the base displacement, and the tracking trend is consistent and the zero error tracking is basically realized in the amplitude.

It is found from Figure 4 that the PSPO needs about $0.3 \mathrm{~s}$ to achieve zero error tracking of the base speed, while the

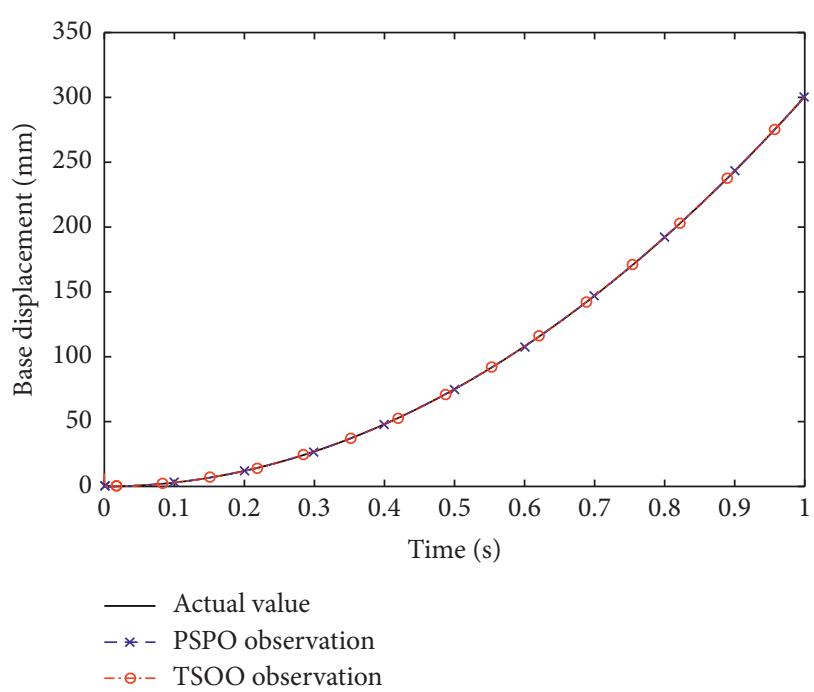

Figure 3: Observation effects of the TSOO and the PSPO on the base displacement.

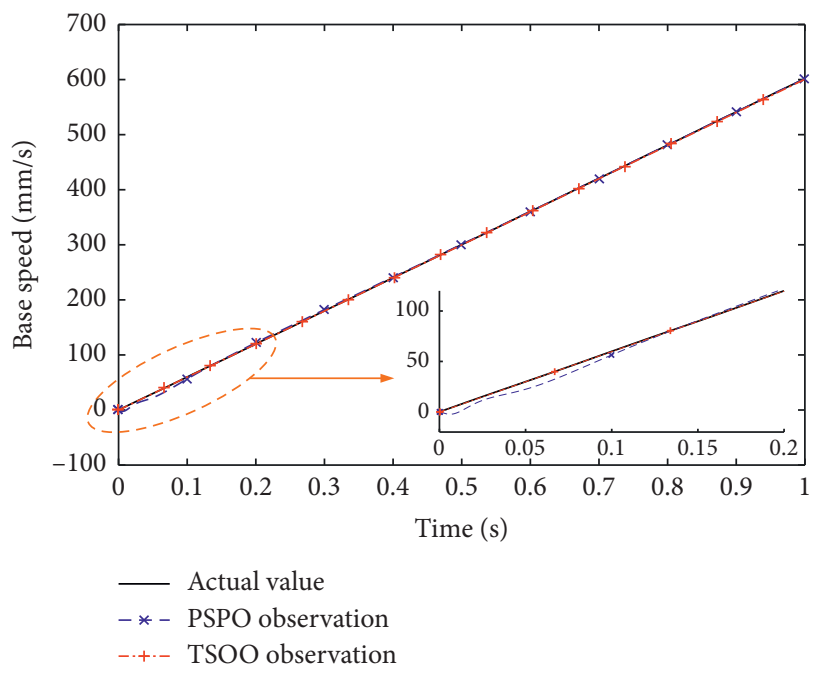

FIGURE 4: Observation effects of the TSOO and the PSPO on the base speed.

designed TSOO has faster convergence speed for the observation error of the base speed. And the special performance is that the tracking trend is consistent and zero error tracking is basically realized in the amplitude. In conclusion, compared with the PSPO, the designed TSOO has a better effect on the observational effect of the base displacement and velocity.

Similarly, under the action of the unit step excitation, the observation effects for the first-order mode coordinates and their derivative of the flexible manipulator are shown in Figures 5 and 6, respectively. And the observation effects for the second-order mode coordinates and their derivative are shown in Figures 7 and 8, respectively.

It is seen from Figures 5 and 6 that after about $0.6 \mathrm{~s}$, both the designed TSOO and the PSPO maintain a high consistency in the tracking trend and the response amplitude of 


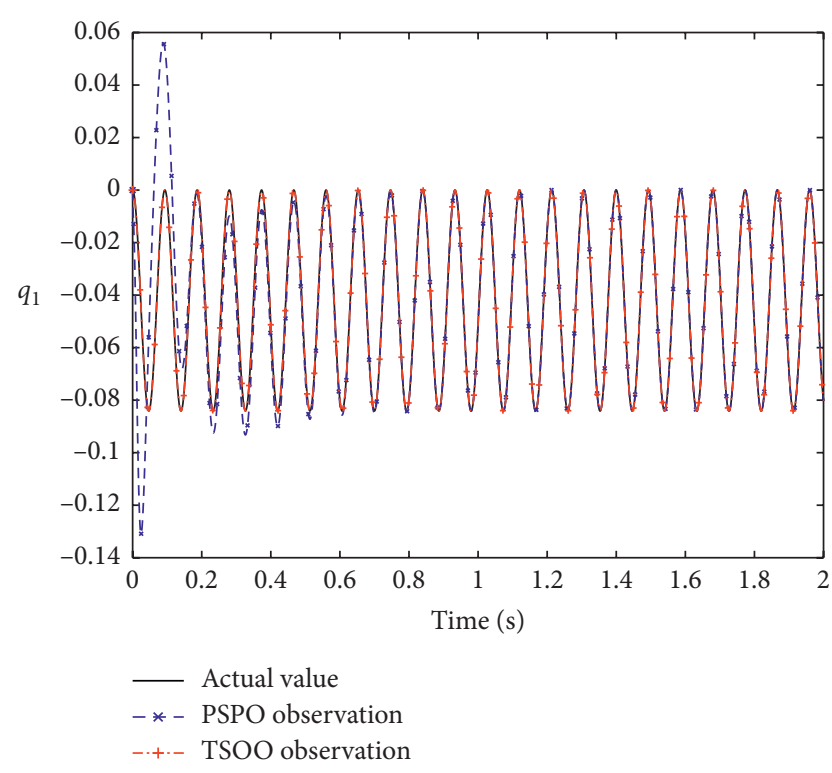

FIGURE 5: Observation effect comparison for the displacement of the first-order mode coordinate.

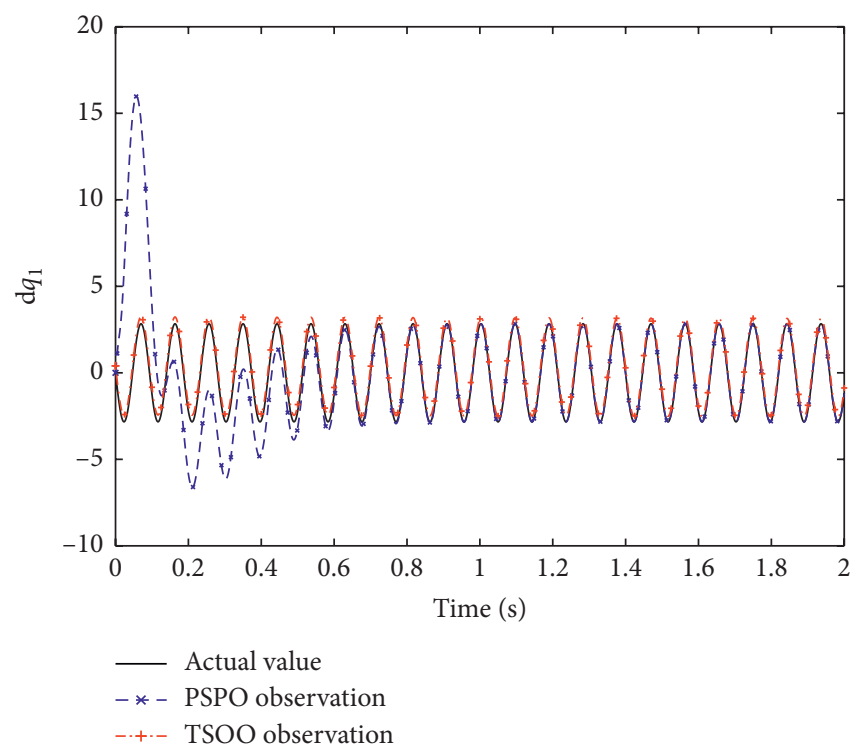

Figure 6: Observation effect comparison for the derivative of the first-order mode coordinate.

the first-order mode and its derivative. In the $0-0.6 \mathrm{~s}$ stage, for the first-order mode or its derivative of the flexible manipulator, the PSPO has a large error in the tracking trend and the response amplitude. Fortunately, the TSOO can accurately track the first-order mode and its derivative from the beginning.

Similarly, as shown in Figures 7 and 8, compared with the PSPO, the TSOO has better observation effects for the second-order mode and its derivative of the flexible manipulator. It takes about $1.3 \mathrm{~s}$ for the PSPO to track the trend and the response amplitude of the second-order mode and its derivative. In conclusion, compared with the PSPO, the designed TSOO has more advantages in tracking the first two mode coordinates and their derivative of the MFMS.

On the other hand, by comparing Figure 5 with Figure 7, it is seen that the response displacement of the second-order mode of the flexible manipulator is negligible compared with its first-order mode response displacement. The premise of the vibration control for the elastic vibration of the MFMS is to ensure that the large-scale vibration caused by the parameter excitation does not occur. And under this premise, the vibration control purpose of the flexible manipulator can be satisfied by effectively suppressing its first-order mode vibration. Thus, in the later control process, only the firstorder mode of the flexible manipulator is necessary to be taken into consideration.

The base motion of the MFMS is set as the trapezoidal velocity motion and the special motion parameters are as follows: a uniformly accelerated motion of $1500 \mathrm{~mm} / \mathrm{s}^{2}$ during 0 to $0.2 \mathrm{~s}$, a uniform velocity motion of $300 \mathrm{~mm} / \mathrm{s}$ during $0.2 \mathrm{~s}$ to $0.8 \mathrm{~s}$, and a uniformly retarded motion of $-1500 \mathrm{~mm} / \mathrm{s}^{2}$ during $0.8 \mathrm{~s}$ to $1.0 \mathrm{~s}$. The control effect of the designed ZV input shaper on the first-order mode of the flexible manipulator under different terminal load conditions is shown in Figure 9.

It is seen that the ZV input shaper can effectively suppress the elastic vibration of the flexible manipulator without the variable load conditions considered. This is because that the ZV input shaper can filter out the frequency components of the input signal which can stimulate the system vibration. However, when there are some variations in the terminal load which is also the system boundary condition, the changes of the vibration mode functions and mode frequencies of the flexible manipulator are inevitable. Because the control parameters of the $\mathrm{ZV}$ input shaper are determined by the structural parameters of the flexible manipulator itself, they cannot adaptively track the changes of the mode functions and the mode frequencies of the flexible manipulator during the control process. Thus, the elastic vibration of the flexible manipulator cannot be effectively suppressed only by the $\mathrm{ZV}$ input shaper under the variable load conditions, which is also obviously shown in Figure 9.

According to Section 3 and the structural parameters of the MFMS, the control gains of the optimal state feedback controller can be determined as $\mathbf{K}_{c}=$ [ $65.71077 .46989 .8637-2.8096$ ]. And based on the optimal state feedback controller and the design method proposed in this paper, the $\mathrm{ZV}$ input shaper is introduced to form the master-slave composite controller. Under the action of the optimal state feedback controller and the designed master-slave composite controller, the control effects for the base displacement of the MFMS are shown in Figure 10, where OSFC means the optimal state feedback controller and MSCC means the designed master-slave composite controller.

It is seen that the optimal state feedback controller and the master-slave composite controller can effectively control the base displacement. However, under almost the same response time, the optimal state feedback controller has a large overshoot and a long adjustment time: the overshoot reaches about $20 \%$ and the adjustment time is about $2 \mathrm{~s}$. 


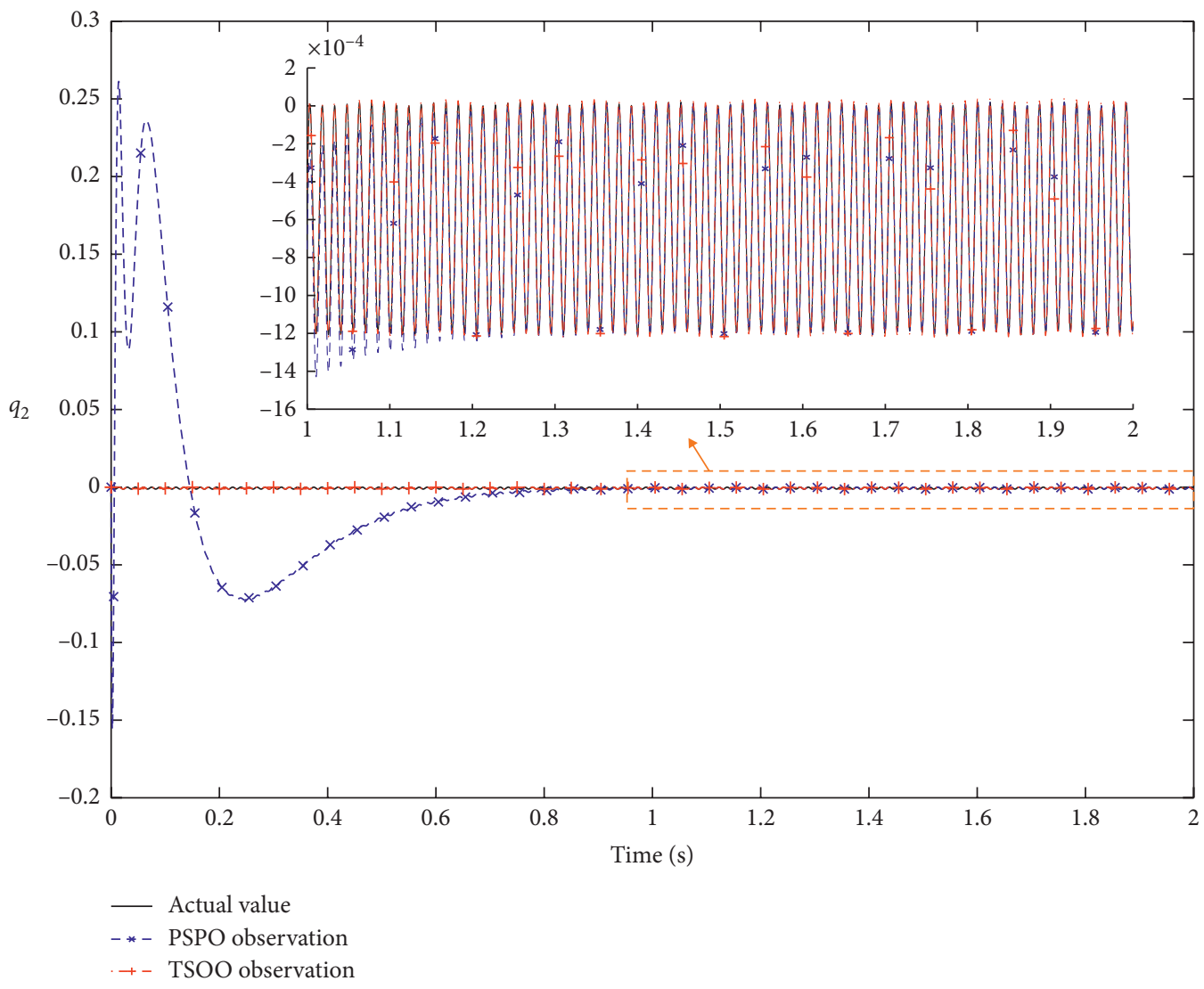

FiguRe 7: Observation effect comparison for the second-order mode coordinate.

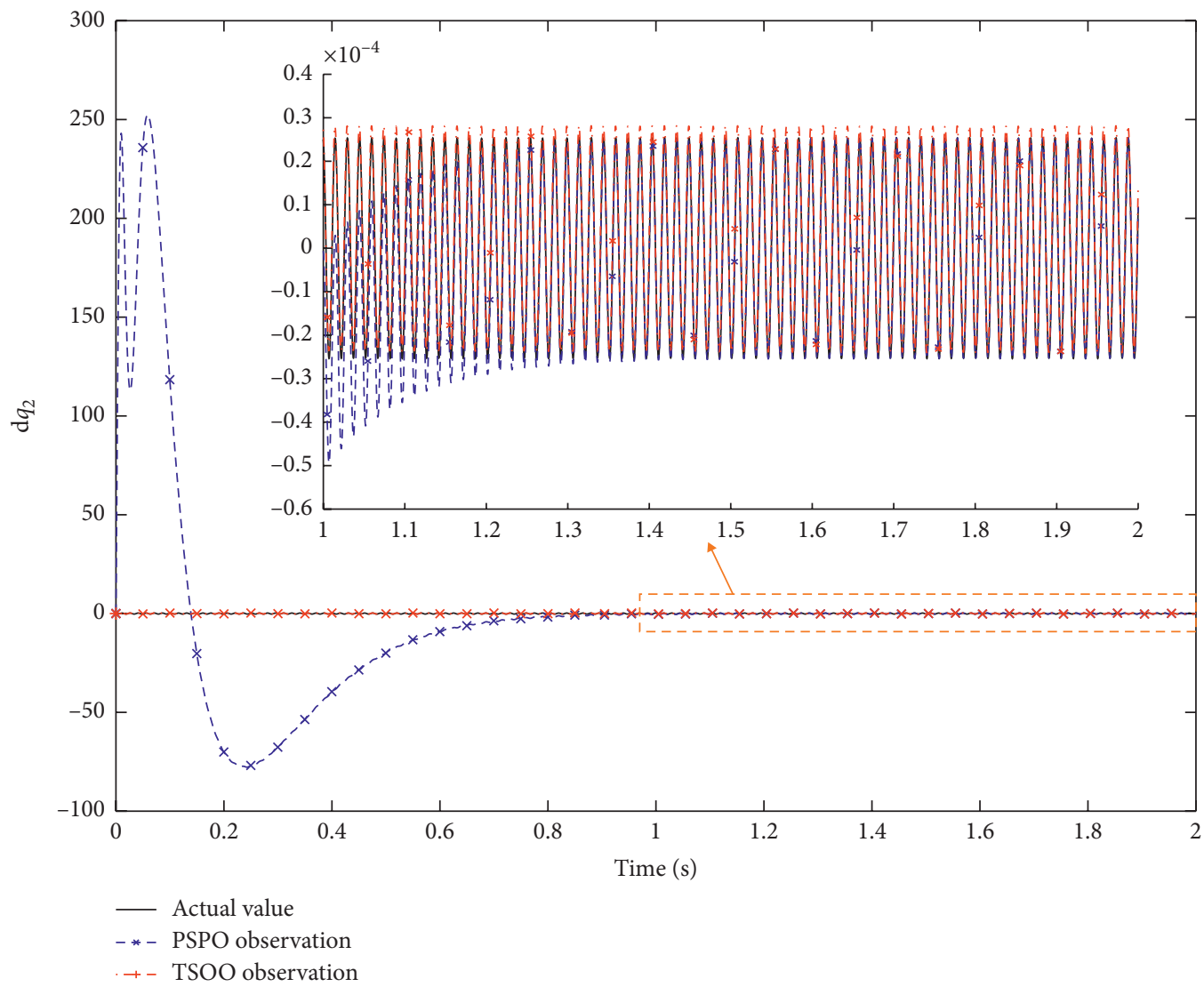

Figure 8: Observation effect comparison for the derivative of the second-order mode coordinate. 


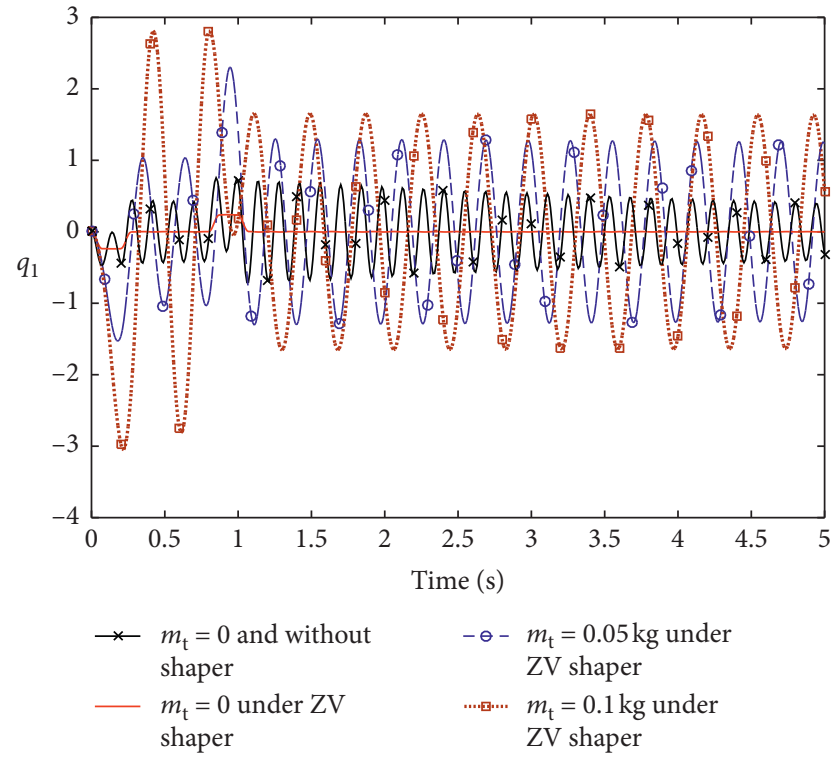

FIGURE 9: Control effects of the ZV input shaper on the first-order mode of the flexible manipulator under different terminal load conditions.

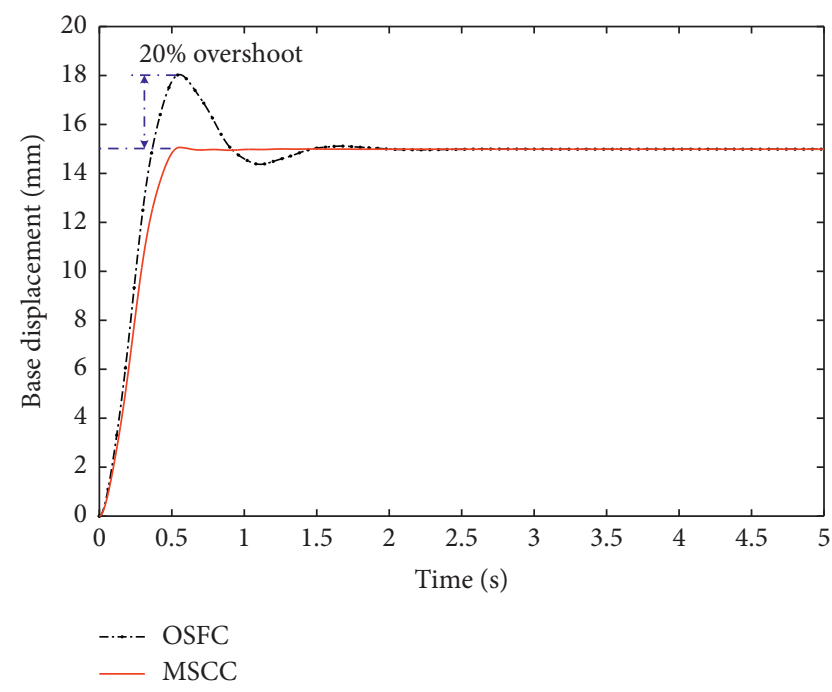

FIGURE 10: Control effects of the master-slave composite controller on the base displacement.

Fortunately, the master-slave composite controller realizes no overshoot, and the adjustment time is about $0.5 \mathrm{~s}$. The reason for this situation is that the contradiction between the response time and the overshoot in the traditional closedloop controller. Fast response time inevitably leads to large overshoot. This problem will inevitably exist in the use process of the optimal state feedback controller which is essentially a closed-loop controller. From the control effect as shown in Figure 10, it is seen that this scheme can effectively improve the control effect of the optimal state feedback controller and achieve the synchronization optimization of the response time and the overshoot.

Figure 11 describes the control effects of the optimal state feedback controller and the master-slave composite

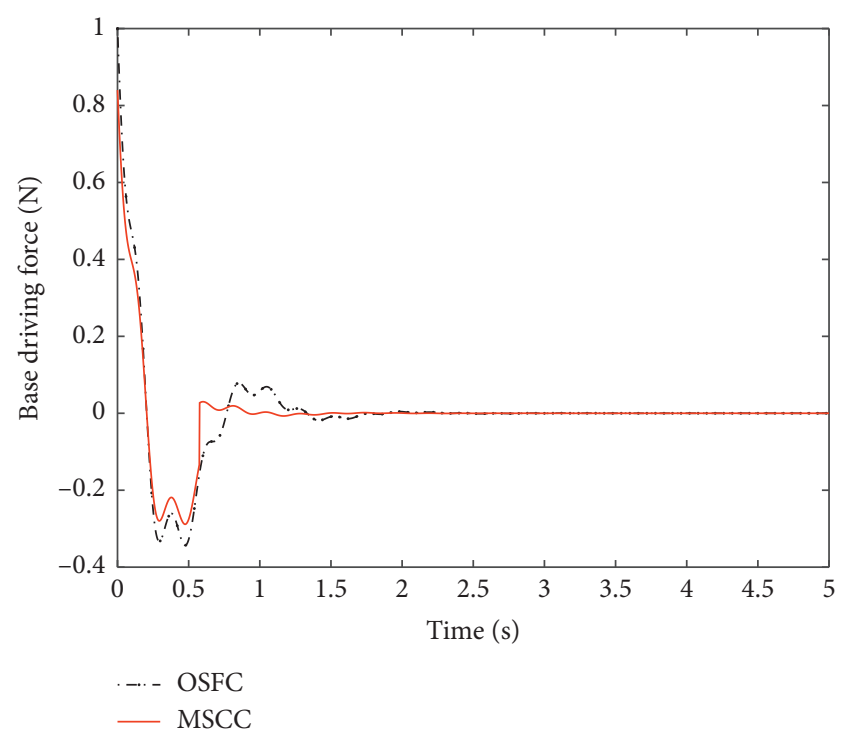

(a)

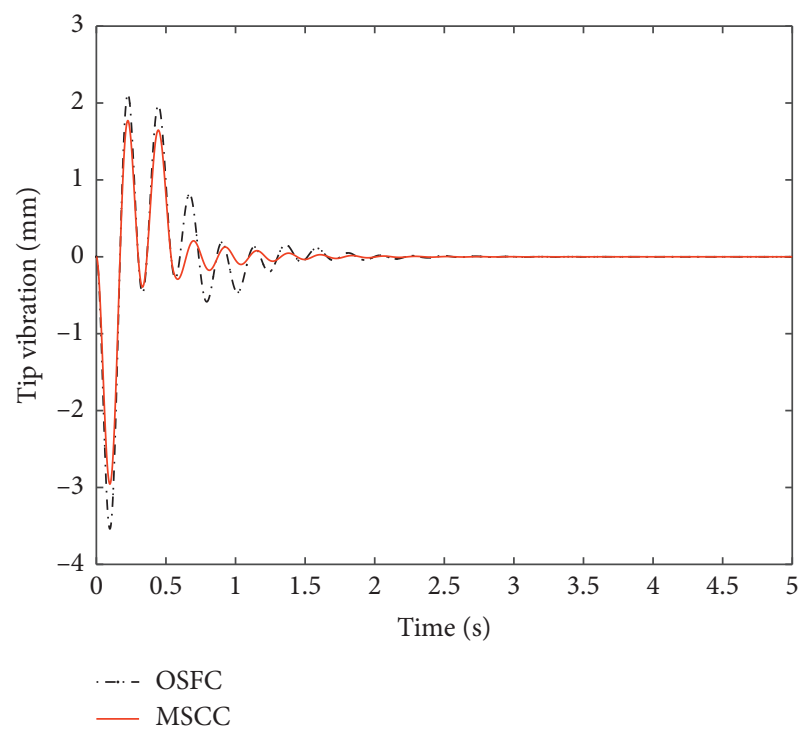

(b)

FIGURE 11: Control effects of the master-slave composite controller and the optimal state feedback controller on the elastic vibration of the MFMS: (a) base driving force and (b) tip vibration.

controller on the elastic vibration of the MFMS. Compared with the optimal state feedback controller, it is found from Figure 11(b) that the master-slave composite controller has better control effect on the system elastic vibration, and the elastic vibration amplitude at the end of the flexible manipulator becomes smaller and the attenuation speed is faster. In addition, Figure 11(a) shows the driving forces of the base under the action of the master-slave composite controller and the optimal state feedback controller. It is seen that the driving force, obtained by the designed masterslave composite controller, comes to stability faster, which means that its adjustment time is shorter. On the other hand, the amplitude change of the driving force obtained by the master-slave composite controller is more stable. Thus, the 


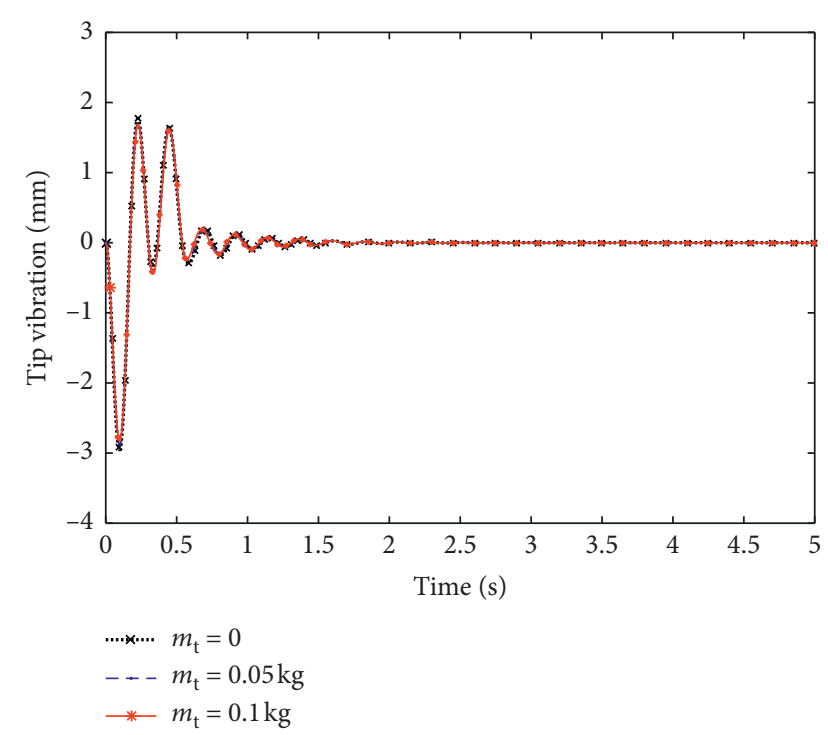

FIGURE 12: Control effects of the master-slave composite controller on the elastic vibration of the MFMS under variable load conditions.

designed master-slave composite controller has a better control effect on the system elastic vibration than the optimal state feedback controller.

Figure 12 describes the control effects of the master-slave composite controller on the elastic vibration of the MFMS under variable load conditions. By comparing Figures 9 and 12 , it is found that the master-slave composite controller can still effectively control the tip vibration of the flexible manipulator under variable load conditions.

In order to further verify the effectiveness of the designed master-slave composite controller, a mechanical model of the mobile flexible manipulator is constructed by ADAMS/ 2013. The mechanical model of the mobile flexible manipulator consists of two parts: one part is the mobile base and the support, and the other is the flexible manipulator. The two parts are concatenated together by fixed constraints. The input is set to the driving force of the mobile base, and the outputs are set to the displacements and speeds of the flexible manipulator tip and the base. Then, the physical model is converted into Simulink subsystem, by the ADAMS/Controls plug-in unit, which is used to establish the combined simulation platform. Similarly, under variable load conditions, the control effects of the master-slave composite controller on the elastic vibration of the flexible manipulator are shown in Figure 13. It is seen that the tip vibration of the flexible manipulator is effectively suppressed by the designed master-slave composite controller on the system combined simulation platform, even under different load conditions.

In summary, with the optimal state feedback controller and the ZV input shaper combined, the masterslave composite controller can effectively realize the complementary advantages and blocking disadvantages of these two controllers on the vibration control of the MFMS. The robustness of the $\mathrm{ZV}$ input shaper under variable load conditions is effectively improved. On the

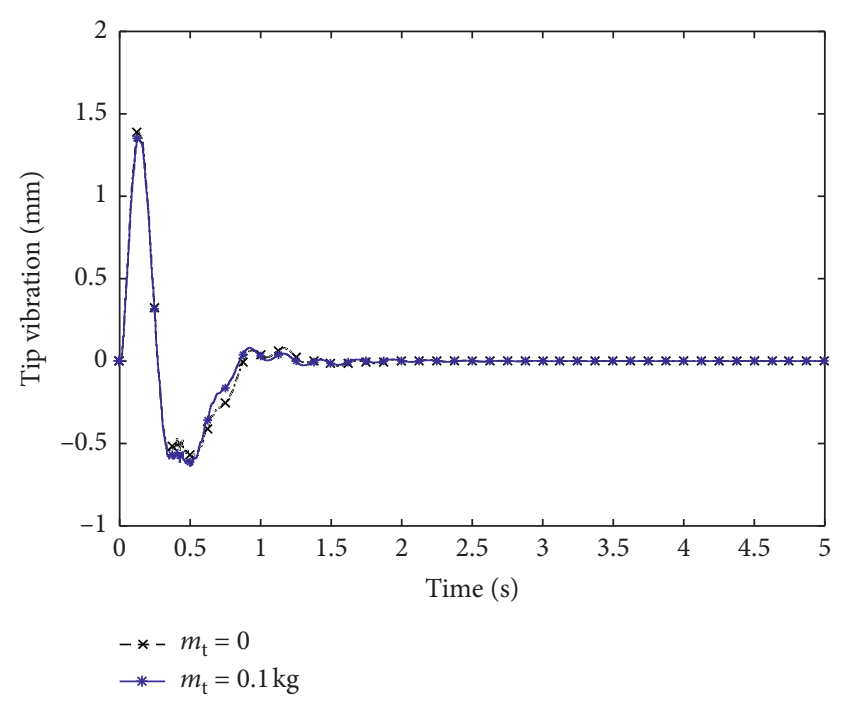

FIGURE 13: Vibration suppression effect of the flexible manipulator under variable load conditions by the combined simulation.

other hand, the synchronization optimization of the overshoot and the response time of the optimal state feedback controller are achieved. Finally, the elastic vibration of the MFMS under variable load conditions can be controlled efficiently and reliably by the designed master-slave composite controller.

\section{Conclusions}

This study focuses on the vibration control of the MFMS. And based on the idea of the master-slave integration, the master-slave composite controller for the elastic vibration of the MFMS is constructed. Simulation experiments results show that

(1) Based on the differential game theory and the constructed two-timescale subsystems of the MFMS, the synchronous optimization for the control gains of the TSOO and the optimal state feedback controller is realized. Compared with the traditional PSPO, the TSOO has great improvement in the observation trend and accuracy for the system state variables.

(2) The design process of the master-slave composite controller is given to effectively integrate the designed optimal state feedback controller and the $\mathrm{ZV}$ input shaper, and the design methods of the related control parameters are determined. The results show that the master-slave composite controller can realize the nonovershoot control of the base displacement under almost the same rise time compared with the optimal state feedback controller, and the adjustment time is reduced from $2 \mathrm{~s}$ to $0.5 \mathrm{~s}$.

(3) Under variable load conditions, the master-slave composite controller can still effectively control the elastic vibration of the MFMS. Through applying the 
control ideas of this paper, the anti-interference ability of the conventional ZV shaper under variable load conditions can be effectively improved.

\section{Data Availability}

The data used to support the findings of this study are available from the corresponding author upon request.

\section{Conflicts of Interest}

The authors declare that there are no conflicts of interest regarding the publication of this paper.

\section{Acknowledgments}

This work was supported by the National Natural Science Foundation of China (no. 51805001), the Anhui Provincial Natural Science Foundation (nos. 1908085QE193 and 1808085QE137), and the Research Starting Fund Project for Introduced Talents of Anhui Polytechnic University (no. 2018YQQ005).

\section{References}

[1] L. Xiao, B. Liao, S. Li, Z. Zhang, L. Ding, and L. Jin, "Design and analysis of FTZNN applied to the real-time solution of a nonstationary Lyapunov equation and tracking control of a wheeled mobile manipulator," IEEE Transactions on Industrial Informatics, vol. 14, no. 1, pp. 98-105, 2018.

[2] Y. Kong, R. Zhang, Y. Jiang, and X. Xia, "A repeatable optimization for kinematic energy system with its mobile manipulator application," Complexity, vol. 2019, Article ID 8642027, 16 pages, 2019.

[3] M. R. Soltanpour and M. H. Khooban, "A particle swarm optimization approach for fuzzy sliding mode control for tracking the robot manipulator," Nonlinear Dynamics, vol. 74, no. 1-2, pp. 467-478, 2013.

[4] S. Haddadin, A. Albu-Schäffer, and G. Hirzinger, "Requirements for safe robots: measurements, analysis and new insights," The International Journal of Robotics Research, vol. 28, no. 11-12, pp. 1507-1527, 2009.

[5] B. Xu and P. C. Zhang, "Composite learning sliding mode control of flexible-link manipulator," Complexity, vol. 2017, Article ID 9430259, 6 pages, 2017.

[6] B. Zi, N. Wang, S. Qian, and K. Bao, "Design, stiffness analysis and experimental study of a cable-driven parallel 3D printer," Mechanism and Machine Theory, vol. 132, pp. 207-222, 2019.

[7] Y. F. Liu, W. Li, X. F. Yang, Y. Q. Wang, M. B. Fan, and G. Ye, "Coupled dynamic model and vibration responses characteristic of a motor-driven flexible manipulator system," Mechanical Sciences, vol. 6, no. 2, pp. 235-244, 2015.

[8] A. Abe, "Trajectory planning for flexible Cartesian robot manipulator by using artificial neural network: numerical simulation and experimental verification," Robotica, vol. 29, no. 5, pp. 797-804, 2011.

[9] Z.-c. Qiu, "Adaptive nonlinear vibration control of a Cartesian flexible manipulator driven by a ballscrew mechanism," Mechanical Systems and Signal Processing, vol. 30, no. 7, pp. 248-266, 2012.

[10] M. O. T. Cole and T. Wongratanaphisan, "A direct method of adaptive FIR input shaping for motion control with zero residual vibration," IEEE/ASME Transactions on Mechatronics, vol. 18, no. 1, pp. 316-327, 2013.

[11] J. Han, Z. Zhu, Y. He, and J. Qi, "A novel input shaping method based on system output," Journal of Sound and Vibration, vol. 335, no. 6, pp. 338-349, 2015.

[12] K. Park, J. Lee, and J. Park, "Torque control of a vehicle with electronic throttle control using an input shaping method," International Journal of Automotive Technology, vol. 14, no. 2, pp. 189-194, 2013.

[13] J. Shan, H. T. Liu, and D. Sun, "Modified input shaping for a rotating single-link flexible manipulator," Journal of Sound and Vibration, vol. 285, no. 1-2, pp. 187-207, 2005.

[14] D. Yuan and T. Chang, "Model reference input shaper design with applications to a high-speed robotic workcell with variable loads," IEEE Transactions on Industrial Electronics, vol. 55, no. 2, pp. 842-851, 2008.

[15] M.-J. Yang, G.-Y. Gu, and L.-M. Zhu, "High-bandwidth tracking control of piezo-actuated nanopositioning stages using closed-loop input shaper," Mechatronics, vol. 24, no. 6, pp. 724-733, 2014.

[16] R. Mar, A. Goyal, V. Nguyen, T. Yang, and W. Singhose, "Combined input shaping and feedback control for doublependulum systems," Mechanical Systems and Signal Processing, vol. 85, pp. 267-277, 2017.

[17] V. Spitsa, A. Alexandrovitz, and E. Zeheb, "Design of a robust state feedback controller for a STATCOM using a zero set concept," IEEE Transactions on Power Delivery, vol. 25, no. 1, pp. 456-467, 2010.

[18] P. M. Vahdati, A. Kazemi, M. H. Amini, and L. Vanfretti, "Hopf bifurcation control of power system nonlinear dynamics via a dynamic state feedback controller-Part I: theory and modeling," IEEE Transactions on Power Systems, vol. 32, no. 4, pp. 3217-3228, 2016.

[19] J. Yao and W. Deng, "Active disturbance rejection adaptive control of hydraulic servo systems," IEEE Transactions on Industrial Electronics, vol. 64, no. 10, pp. 8023-8032, 2017.

[20] P. M. Vahdati, L. Vanfretti, M. H. Amini, and A. Kazemi, "Hopf bifurcation control of power systems nonlinear dynamics via a dynamic state feedback controller-Part II: performance evaluation," IEEE Transactions on Power Systems, vol. 32, no. 4, pp. 3229-3236, 2017.

[21] Y.-J. Liu and S. Tong, "Barrier Lyapunov functions-based adaptive control for a class of nonlinear pure-feedback systems with full state constraints," Automatica, vol. 64, pp. 70-75, 2016.

[22] H. Yang, J. Liu, and X. Lan, "Observer design for a flexiblelink manipulator with PDE model," Journal of Sound and Vibration, vol. 341, pp. 237-245, 2015.

[23] J. Yao and W. Deng, "Active disturbance rejection adaptive control of uncertain nonlinear systems: theory and application," Nonlinear Dynamics, vol. 89, no. 3, pp. 1611-1624, 2017.

[24] S. K. Dwivedy and P. Eberhard, "Dynamic analysis of flexible manipulators, a literature review," Mechanism and Machine Theory, vol. 41, no. 7, pp. 749-777, 2006.

[25] H. N. Rahimi and M. Nazemizadeh, "Dynamic analysis and intelligent control techniques for flexible manipulators: a review," Advanced Robotics, vol. 28, no. 2, pp. 63-76, 2014.

[26] C. Yang, Y. Xu, L. Zhou, and Y. Sun, "Model-free composite control of flexible manipulators based on adaptive dynamic programming," Complexity, vol. 2018, Article ID 9720309, 9 pages, 2018.

[27] A. E. Firoozabadi, S. Ebrahimi, and J. M. Font-Llagunes, “A comparative study of elastic motions in trajectory tracking of 
flexible RPR planar manipulators moving with high speed," Robotica, vol. 35, no. 7, pp. 1-18, 2016.

[28] C. Wang and L. Wu, "Chaotic vibration prediction of a freefloating flexible redundant space manipulator," Shock and Vibration, vol. 2016, Article ID 6015275, 12 pages, 2016.

[29] K. Alipour, P. Zarafshan, and A. Ebrahimi, "Dynamics modeling and attitude control of a flexible space system with active stabilizers," Nonlinear Dynamics, vol. 84, no. 4, pp. 2535-2545, 2016.

[30] Z. Hong and L. Chen, "Active vibration control and fuzzy control of free-floating space flexible manipulator based on singular perturbation theory," Journal of Mechanical Engineering, vol. 46, no. 7, pp. 35-41, 2010.

[31] M. C. Pai, "Closed-loop input shaping control of vibration in flexible structures via adaptive sliding mode control," Shock and Vibration, vol. 19, no. 2, pp. 221-233, 2015.

[32] W. P. Li, B. Luo, and H. Huang, "Active vibration control of flexible joint manipulator using input shaping and adaptive parameter auto disturbance rejection controller," Journal of Sound and Vibration, vol. 363, pp. 97-125, 2016.

[33] N. X. Hong and C. Li, "Design of optimal observer and optimal feedback controller based on differential game theory," Acta Automatica Sinica, vol. 32, no. 5, pp. 807-812, 2006.

[34] H. Zhang, J. Zhang, G.-H. Yang, and Y. Luo, "Leader-based optimal coordination control for the consensus problem of multi-agent differential games via fuzzy adaptive dynamic programming," IEEE Transactions on Fuzzy Systems, vol. 23, no. 1, pp. 152-163, 2014.

[35] K.-H. Rew, C.-W. Ha, and K.-S. Kim, "An impulse-time perturbation approach for enhancing the robustness of extrainsensitive input shapers," Automatica, vol. 49, no. 11, pp. 3425-3431, 2013.

[36] M. Hromcik and T. Vyhlidal, "Inverse feedback shapers for coupled multibody systems," IEEE Transactions on Automatic Control, vol. 62, no. 9, pp. 4804-4810, 2017.

[37] J.-y. Ju, W. Li, Y. Liu, and C. Zhang, "Master-slave integrated control for the transverse vibration of a translational flexible manipulator based on input shaping and state feedback," Shock and Vibration, vol. 2019, Article ID 8419591, 13 pages, 2019.

[38] E. Pereira, S. S. Aphale, V. Feliu, and S. O. R. Moheimani, "Integral resonant control for vibration damping and precise tip-positioning of a single-link flexible manipulator," IEEE/ ASME Transactions on Mechatronics, vol. 16, no. 2, pp. 232240, 2011. 


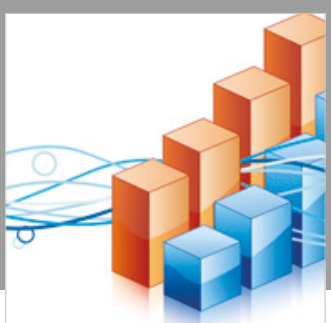

Advances in

Operations Research

\section{-n-m}
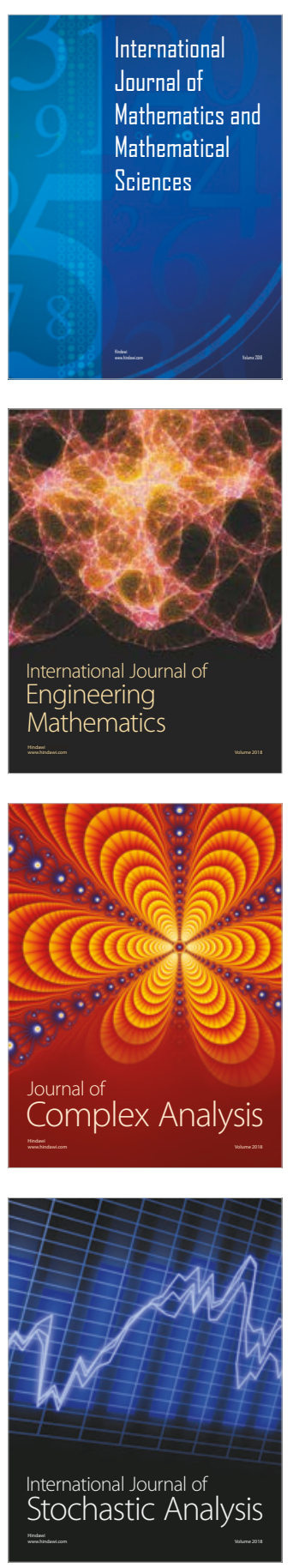
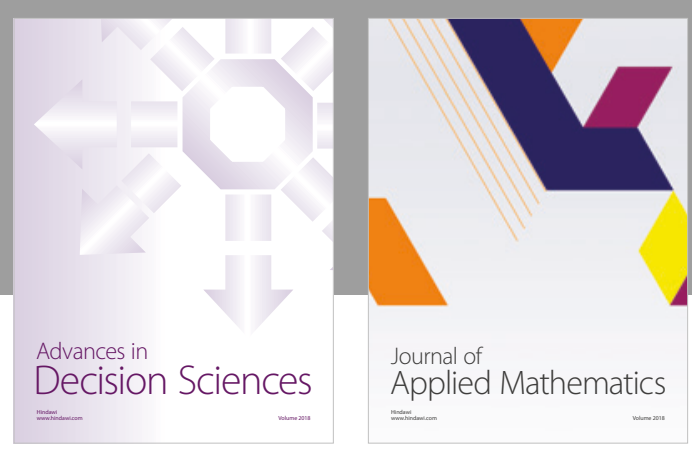

Journal of

Applied Mathematics
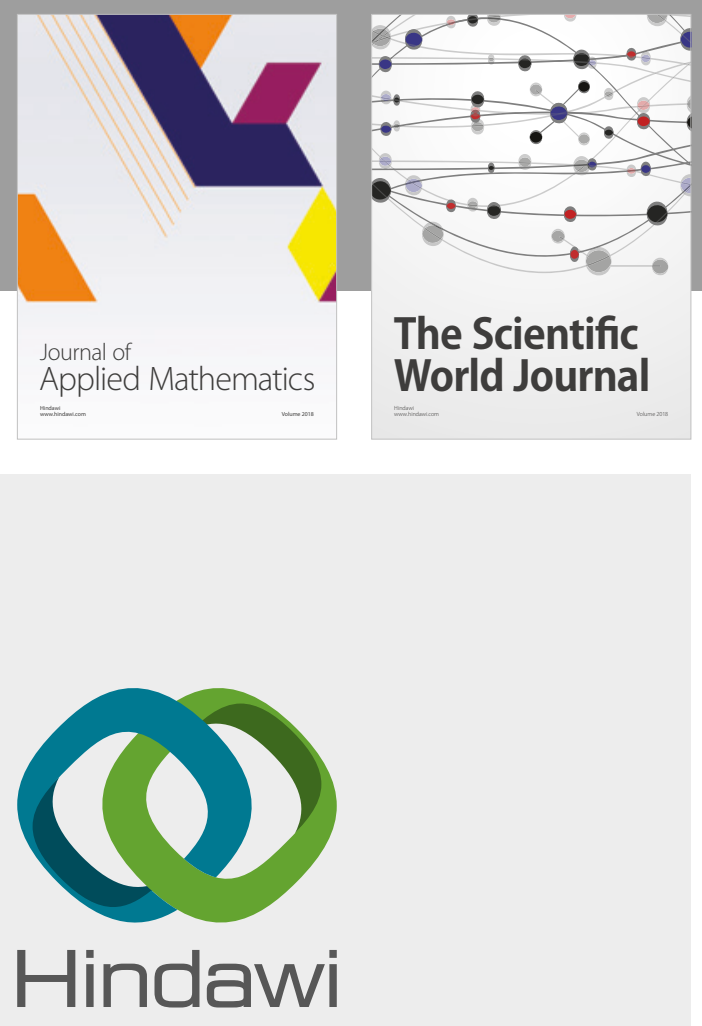

Submit your manuscripts at

www.hindawi.com

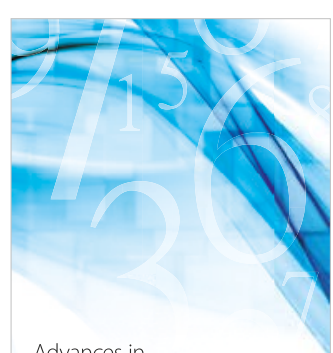

Advances in
Numerical Analysis
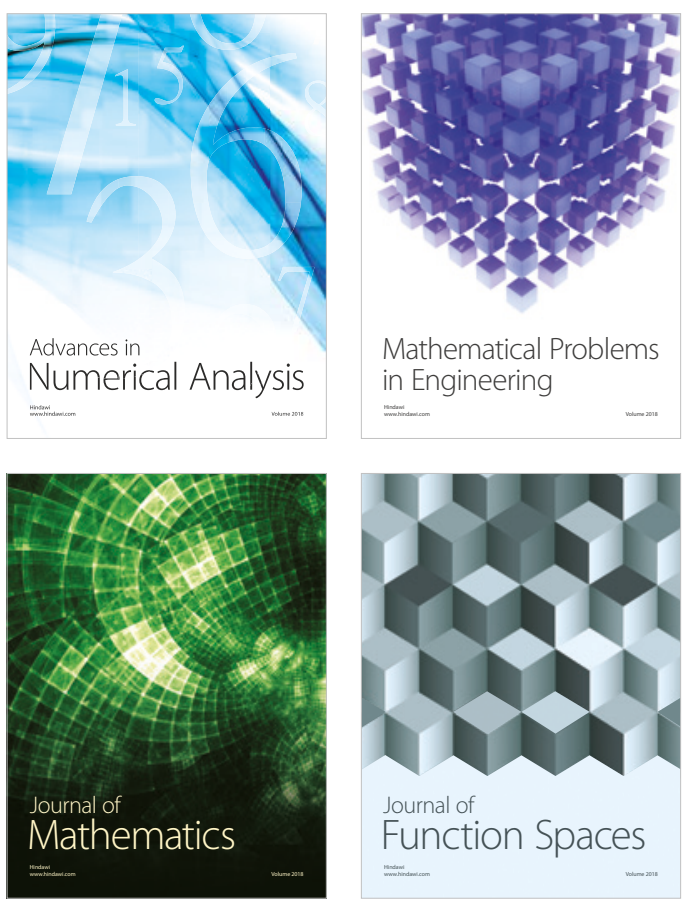

Mathematical Problems in Engineering

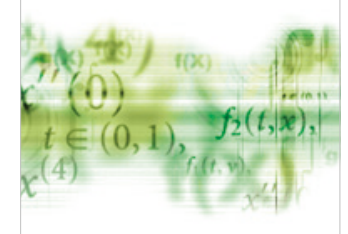

International Journal of

Differential Equations

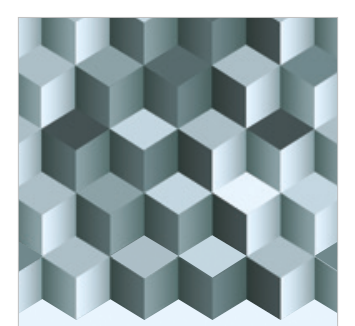

Journal of

Function Spaces
The Scientific

World Journal

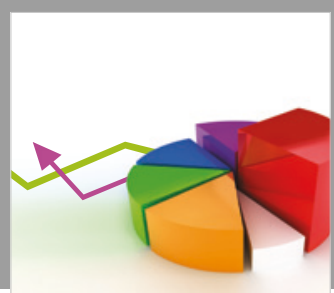

Journal of

Probability and Statistics
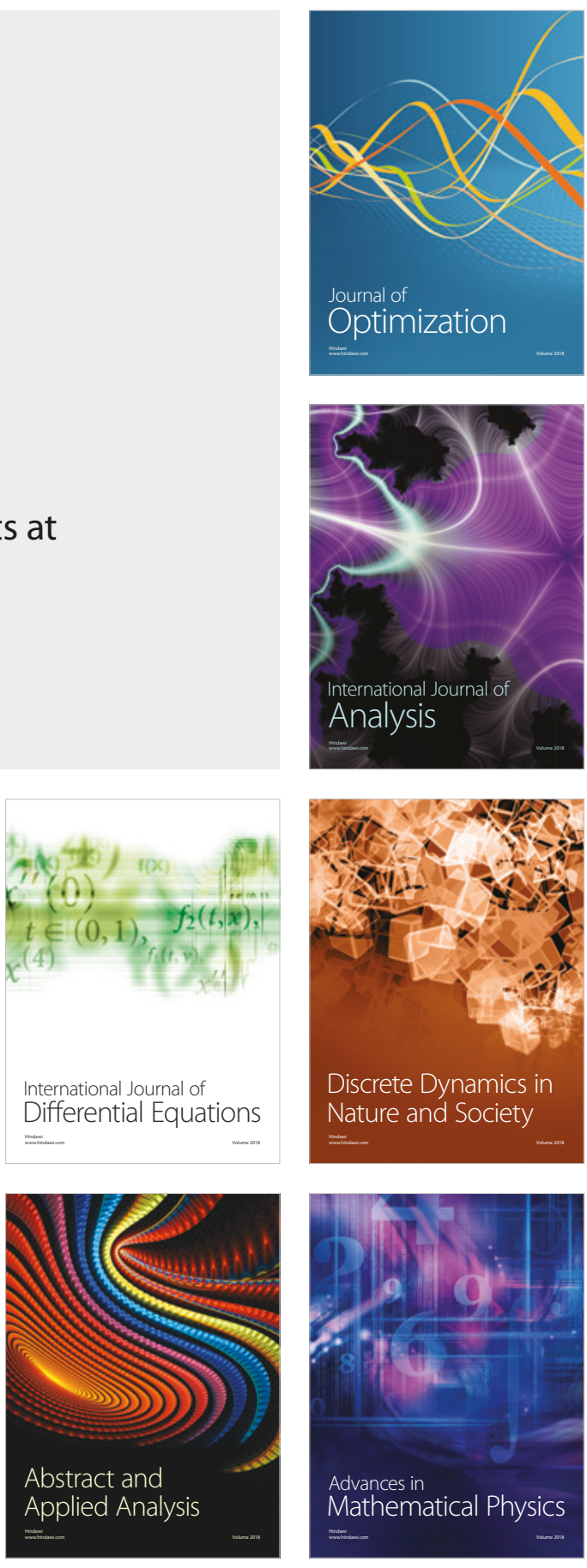